\title{
On the Homotopy Types of the Groups of Equivariant Diffeomorphisms
}

By

Kōjun $\mathrm{ABE}^{*}$

\section{$\S 0$. Introduction}

The purpose of this paper is to study the homotopy type of the group of the equivariant diffeomorphisms of a closed connected smooth $G$-manifold $M$, when $G$ is a compact Lie group and the orbit space $M / G$ is homeomorphic to a unit interval $[0,1]$.

Let $\operatorname{Diff}_{G}^{\infty}(M)_{0}$ denote the group of equivariant $C^{\infty}$ diffeomorphisms of the $G$-manifold $M$ which are $G$-isotopic to the identity, endowed with $C^{\infty}$ topology. If $M / G$ is homeomorphic to $[0,1]$, then $M$ has two or three orbit types $G / H, G / K_{0}$ and $G / K_{1}$. We can choose the isotropy subgroups $H, K_{0}$, $K_{1}$ satisfying $H \subset K_{0} \cap K_{1}$. Moreover the $G$-manifold structure of $M$ is determined by an element $\eta$ of a factor group $N(H) / H$, where $N(H)$ is the normalizer of $H$ in $G$ (see $\S 1)$. Let $\Omega\left(N(H) / H ;\left(N(H) \cap N\left(K_{0}\right)\right) / H,(N(H) \cap\right.$ $\left.\left.N\left(\eta K_{1} \eta^{-1}\right)\right) / H\right)_{0}$ denote the connected component of the identity of the space of paths $a:[0,1] \rightarrow N(H) / H$ satisfying $a(0) \in\left(N(H) \cap N\left(K_{0}\right)\right) / H$ and $a(1) \in(N(H) \cap$ $\left.N\left(\eta K_{1} \eta^{-1}\right)\right) / H$.

Theorem. Diff $G_{G}^{\infty}(M)_{0}$ has the same homotopy type as the path space $\Omega\left(N(H) / H ;\left(N(H) \cap N\left(K_{0}\right)\right) / H,\left(N(H) \cap N\left(\eta K_{1} \eta^{-1}\right)\right) / H\right)_{0}$.

The paper is organized as follows. In Section 1, we study the $G$-manifold structure of $M$ and give a differentiable structure of $M / G$ such that the functional structure of $M / G$ is induced from that of $M$. This differentiable structure is important to study the structure of $\operatorname{Diff}_{G}^{\infty}(M)_{0}$. In Section 2, we define a group homomorphism $P: \operatorname{Diff}_{G}^{\infty}(M)_{0} \rightarrow \operatorname{Diff}^{\infty}[0,1]_{0}$ and prove that $P$ is a continuous homomorphism between topological groups. In Section 3, we

Communicated by N. Shimada, April 10, 1979.

* Department of Mathematics, Shinshu University. 
prove that there exists a global continuous section of $P$ and $\operatorname{Ker} P$ is a deformation retract of $\operatorname{Diff}_{G}^{\infty}(M)_{0}$. In Section 4, we study the group structure of Ker $P$. In Section 5 and Section 6, we prove our Theorem.

\section{§1. G-Manifold Structure of $M$ and the Functional Structure of $M / G$}

In this paper we assume that all manifolds and all actions are differentiable of class $C^{\infty}$.

In this section we study the $G$-manifold structure of $M$. First we see that it is sufficient for us to consider $\eta=1$ (see Lemma 1.1). Next we give a differentiable structure of $M / G$ such that the functional structure of $M / G$ is induced from that of $M$ (see Lemma 1.2).

Let $M$ be a closed connected smooth $G$-manifold such that $M / G$ is homeomorphic to $[0,1]$. We denote this homeomorphism by $f$. Let $\pi: M \rightarrow M / G$ be the natural projection. Put $M_{0}=(f \circ \pi)^{-1}([0,1 / 2])$ and $M_{1}=(f \circ \pi)^{-1}([1 / 2$, 1]). Let $x_{i}$ be a point of $M$ with $f\left(\pi\left(x_{i}\right)\right)=i$ for $i=0,1$. Then $M_{i}$ is a $G$ invariant closed tubular neighborhood of the orbit $G\left(x_{i}\right)$ (c.f. G. Bredon [3, Chapter VI, §6]). Moreover $M$ is equivariantly diffeomorphic to a union of the $G$-manifolds $M_{0}$ and $M_{1}$ such that their boundaries are identified under a $G$-diffeomorphism $\eta^{\prime}: \partial M_{0} \rightarrow \partial M_{1}$. Let $V_{i}$ be a normal vector space of $G\left(x_{i}\right)$ at $x_{i}$ and $K_{i}$ be the isotropy subgroup of $x_{i}$ for $i=0,1$. Then $V_{i}$ is a representation space of $K_{i}$. From the differentiable slice theorem, $M_{i}$ is equivariantly diffeomorphic to a smooth $G$-bundle $G \times{ }_{K_{i}} D\left(V_{i}\right)$ which is associated to the principal $K_{i}$ bundle $\pi_{i}: G \rightarrow G / K_{i}$, where $D\left(V_{i}\right)$ is a unit disc in $V_{i}$.

Let $H$ be a principal isotropy subgroup of the $G$-manifold $M$. We can assume that $H$ is a subgroup of $K_{0} \cap K_{1}$. Let $e_{i} \in S\left(V_{i}\right)$ be a point such that the isotropy subgroup of $e_{i}$ is $H$ for $i=0,1$, where $S\left(V_{i}\right)$ is a unit sphere in $V_{i}$. There exists a $G$-diffeomorphism $h_{i}: G / H \rightarrow G \times_{K_{i}} S\left(V_{i}\right)$ given by $h_{i}(g H)=\left[g, e_{i}\right]$, $i=0,1$. Then we have a $G$-diffeomorphism

$$
\eta^{\prime \prime}: G / H \stackrel{h_{0}}{\longrightarrow} G \times_{K_{0}} S\left(V_{0}\right)=\partial M_{0} \stackrel{\eta^{\prime}}{\longrightarrow} \partial M_{1}=G \times_{K_{1}} S\left(V_{1}\right) \stackrel{h_{1}-1}{\longrightarrow} G / H .
$$

Since any $G$-map $G / H \rightarrow G / H$ is given by a right translation of an element of $N(H) / H, \eta^{\prime \prime}$ defines an element $\eta \in N(H) / H$.

Put $x_{i}^{\prime}=\eta \cdot x_{i}$. Then the isotropy subgroup $K_{1}^{\prime}$ of $x_{1}^{\prime}$ is $\eta K_{1} \eta^{-1}$. Let $V_{1}^{\prime}$ be a normal vector space of the orbit $G\left(x_{1}^{\prime}\right)=G\left(x_{i}\right)$ at $x_{1}^{\prime}$. Put $e_{1}^{\prime}=(d \eta)_{x_{1}}\left(e_{1}\right) \in$ $S\left(V_{1}^{\prime}\right)$. There exists a $G$-diffeomorphism $u: G \times_{K_{1}} D\left(V_{1}\right) \rightarrow G \times_{K_{1}^{\prime}} D\left(V_{1}^{\prime}\right)$ given 
by $u([g, v])=\left[g \eta^{-1}, \eta \cdot v\right]$. Then $\left(u \circ \eta^{\prime}\right)\left(\left[g, e_{0}\right]\right)=u\left(\left[g \eta, e_{1}\right]\right)=\left[g, e_{1}^{\prime}\right]$ for $[g, v]$ $\in G \times{ }_{K_{0}} S\left(V_{0}\right)$. Therefore $M$ is equivariantly diffeomorphic to a union of the $G$-bundles $G \times_{K_{0}} D\left(V_{0}\right)$ and $G \times_{K_{1}^{\prime}} D\left(V_{1}^{\prime}\right)$ such that their boundaries are identified under a $G$-diffeomorphism $u \circ \eta^{\prime}$. Now we have

Lemma 1.1. Let $M$ be a closed connected smooth G-manifold such that $M / G$ is homeomorphic to $[0,1]$. Then $M$ has two or three orbit types $G / H$, $G / K_{0}$ and $G / K_{1}$ with $H \subset K_{0} \cap K_{1}$, and there exist representation spaces $V_{i}$, $i=0,1$, of $K_{i}$ such that $M$ is equivariantly diffeomorphic to a union of $G$ bundles $G \times{ }_{K_{0}} D\left(V_{0}\right)$ and $G \times{ }_{K_{1}} D\left(V_{1}\right)$ with their boundaries identified under a G-diffeomorphism $\eta: G \times{ }_{K_{0}} S\left(V_{0}\right) \rightarrow G \times{ }_{K_{1}} S\left(V_{1}\right)$. Moreover we may assume that $\eta\left(\left[g, e_{0}\right]\right)=\left[g, e_{1}\right]$, where $e_{i}$ is a point of $S\left(V_{i}\right)$ such that the isotropy subgroup of $e_{i}$ is $H$ for $i=0,1$.

Hereafter we shall assume that $M$ is a $G$-manifold as in Lemma 1.1. Let $\xi:[0,1] \rightarrow R$ be a smooth function such that

$$
\begin{aligned}
& \xi(r)=r^{2} \text { for } 0 \leq r \leq 1 / 2, \\
& \xi^{\prime}(r)>0 \text { for } 0<r \leq 1 \text { and } \\
& \xi(r)=r-1 / 2 \text { for } 7 / 8<r \leq 1
\end{aligned}
$$

Let $\theta: M=G \times_{K_{0}} D\left(V_{0}\right) \bigcup_{\eta} G \times_{K_{1}} D\left(V_{1}\right) \rightarrow[0,1]$ be a map given by

$$
\begin{array}{lll}
\theta([g, v])=\xi(\|v\|) & \text { for } & {[g, v] \in G \times \times_{K_{0}} D\left(V_{0}\right),} \\
\theta([g, v])=1-\xi(\|v\|) & \text { for } & {[g, v] \in G \times \times_{K_{1}} D\left(V_{1}\right) .}
\end{array}
$$

Since $\theta$ is a $G$-map, there exists a map $\phi: M / G \rightarrow[0,1]$ such that $\phi \circ \pi=\theta$. It is easy to see that $\phi$ is a homeomorphism. We give a differentiable structure of $M / G$ by $\phi$.

Lemma 1.2. In the above situation, we have

(1) $\theta$ is a smooth map,

(2) there exists a G-diffeomorphism $\alpha: \theta^{-1}((0,1)) \rightarrow G / H \times(0,1)$ such that $\theta \circ \alpha^{-1}$ is the projection on the second factor, and

(3) $f: M / G \rightarrow R$ is smooth if and only if $f \circ \pi: M \rightarrow R$ is smooth.

Proof. (1) Let $\alpha_{i}: G \times{ }_{K_{i}}\left(D\left(V_{i}\right)-0\right) \rightarrow G / H \times(0,1]$ be a map given by $\alpha_{i}\left(\left[g, r e_{i}\right]\right)=(g H, r)$ for $g \in G$ and $r \in(0,1](i=0,1)$. Then it is easy to see that $\alpha_{i}$ is a $G$-diffeomorphism. Since $\alpha_{1} \circ \eta=\alpha_{0}$ on $G \times{ }_{K_{0}} S\left(V_{0}\right)$, the composition $\beta: \theta^{-1}((0,1))=G \times_{K_{0}}\left(D\left(V_{0}\right)-0\right) \cup G \times_{K_{1}}\left(D\left(V_{1}\right)-0\right) \stackrel{\alpha_{0} \cup \alpha_{1}}{\longrightarrow} G / H \times(0,1] \cup_{1_{G / H} \times 1}$ $G / H \times(0,1]=G / H \times(0,2)$ is a $G$-diffeomorphism. Note that 


$$
\left(\theta \circ \beta^{-1}\right)(g H, r)= \begin{cases}\xi(r) & \text { for } 0<r \leq 1 \\ 1-\xi(2-r) & \text { for } 1 \leq r \leq 2\end{cases}
$$

Thus $\theta \circ \beta^{-1}$ is a smooth map, and $\theta$ is a smooth map on $\theta^{-1}((0,1))$. From the definition, $\theta$ is a smooth map on $\theta^{-1}(r)$ for $r \neq 1 / 2$. Therefore $\theta$ is a smooth map.

(2) Let $\bar{\theta}:(0,2) \rightarrow(0,1)$ be a smooth map given by

$$
\bar{\theta}(r)= \begin{cases}\xi(r) & \text { for } 0<r \leq 1 \\ 1-\xi(2-r) & \text { for } 1 \leq r<2 .\end{cases}
$$

Since $\bar{\theta}^{\prime}(r)>0$ for $0<r<2, \bar{\theta}$ is a diffeomorphism. Let $\alpha: \theta^{-1}((0,1)) \rightarrow G / H$ $\times(0,1)$ be a $G$-diffeomorphism given by $\alpha=(1, \bar{\theta}) \circ \beta$. Then $\left(\theta \circ \alpha^{-1}\right)(g H, r)$ $=\left(\theta \circ \beta^{-1}\right)\left(g H, \bar{\theta}^{-1}(r)\right)=r$, and $\theta \circ \alpha^{-1}$ is the projection on the second factor.

(3) Let $f: M / G \rightarrow R$ be a function such that $f \circ \pi: M \rightarrow R$ is smooth. We shall prove that $f \circ \phi^{-1}:[0,1] \rightarrow R$ is smooth. Since

$$
\left(f \circ \pi \circ \alpha^{-1}\right)(g H, r)=\left(f \circ \phi^{-1} \circ \theta \circ \alpha^{-1}\right)(g H, r)=\left(f \circ \phi^{-1}\right)(r) \text { for } 0<r<1,
$$

$f \circ \phi^{-1}$ is smooth on $(0,1)$. Let $i_{0}: D_{1 / 2}\left(V_{0}\right)=\left\{v \in D\left(V_{0}\right) ;\|v\| \leq 1 / 2\right\} \rightarrow G \times{ }_{K_{0}}$ $D\left(V_{0}\right)$ be an inclusion given by $i_{0}(v)=[1, v]$. Note that $\left(\theta \circ i_{0}\right)(v)=\|v\|^{2}$ for $v \in D_{1 / 2}\left(V_{0}\right)$. By Corollary 5.3 of $G$. Bredon [3, Chapter VI, $\left.\S 5\right], f \circ \phi^{-1}$ is smooth if and only if $\left(f \circ \phi^{-1}\right) \circ\left(\theta \circ i_{0}\right)$ is smooth. Since $\left(f \circ \phi^{-1}\right) \circ\left(\theta \circ i_{0}\right)=f \circ \pi \circ i_{0}$, which is smooth, then $f \circ \phi^{-1}$ is smooth on $[0,1 / 4]$. Similarly we can prove that $f \circ \phi^{-1}$ is smooth on $[3 / 4,1]$. Since $\left(f \circ \phi^{-1}\right)(r)=\left(f \circ \phi^{-1} \circ \theta \circ \alpha^{-1}\right)(1 H, r)$ $=\left(f \circ \pi \circ \alpha^{-1}\right)(1 H, r)$ for $0<r<1, f \circ \phi^{-1}$ is smooth on $(0,1)$. This completes the proof of Lemma 1.2.

Remark 1.3. Lemma 1.2 is essentially proved by G. Bredon [3, Chapter VI, §5], and (3) implies that the functional structure of $M / G$ is induced from that of $M$.

\section{§2. On the Group Homomorphism $\boldsymbol{P}$}

In this section we shall define a group homomorphism $P: \operatorname{Diff}_{G}^{\infty}(M)_{0} \rightarrow$ $\operatorname{Diff}^{\infty}[0,1]$, and we shall prove $P$ is continuous.

We shall identify the orbit space $M / G$ with $[0,1]$ by the homeomorphism $\phi$ in $\S 1$, therefore the projection $\pi: M \rightarrow M / G$ is identified with the smooth map $\theta: M \rightarrow[0,1]$. Let $h: M \rightarrow M$ be a $G$-diffeomorphism of $M$ which is $G$-isotopic to the identity $1_{M}$, and let $f:[0,1] \rightarrow[0,1]$ be the orbit map of $h$. Since $f \circ \pi$ $=\pi \circ h$ is a smooth map, $f$ is a smooth map by Lemma $1.2(3)$. Similarly the 
inverse map $f^{-1}$ of $f$ is smooth, and $f$ is a diffeomorphism. Then there exists an abstract group homomorphism $P: \operatorname{Diff}_{G}^{\infty}(M)_{0} \rightarrow \operatorname{Diff}^{\infty}[0,1]$ which is given by $P(h)=f$, where $\operatorname{Diff}^{\infty}[0,1]$ is the group of $C^{\infty}$ diffeomorphisms of $[0,1]$, endowed with $C^{\infty}$ topology.

Proposition 2.1. $P: \operatorname{Diff}_{G}^{\infty}(M)_{0} \rightarrow \operatorname{Diff}^{\infty}[0,1]$ is a continuous homomorphism of topological groups.

Let $C^{\infty}\left(M_{1}, M_{2}\right)$ denote the set of all smooth maps from a compact smooth manifold $M_{1}$ to a smooth manifold $M_{2}$, endowed with $C^{\infty}$ topology. Before the proof of Proposition 2.1, we begin with some lemmas.

Lemma 2.2. Let $M_{i}$ be a compact smooth manifold and $N_{i}$ be a smooth manifold for $i=1,2$. Then we have:

(1) Let $\phi: N_{1} \rightarrow N_{2}$ be a smooth map, and let $\phi_{*}: C^{\infty}\left(M_{1}, N_{1}\right) \rightarrow C^{\infty}\left(M_{1}\right.$, $N_{2}$ ) be a map which is given by $\phi_{*}(f)=\phi \circ f$. Then $\phi_{*}$ is continuous.

(2) Let $\phi: M_{1} \rightarrow M_{2}$ be a smooth map, and let $\phi^{*}: C^{\infty}\left(M_{2}, N_{1}\right) \rightarrow C^{\infty}\left(M_{1}\right.$, $N_{1}$ ) be a map which is given by $\phi^{*}(f)=f \circ \phi$. Then $\phi^{*}$ is continuous.

(3) Let $\phi: M_{1} \rightarrow N_{2}$ be a smooth map and let $\phi_{\#}: C^{\infty}\left(M_{1}, N_{1}\right) \rightarrow C^{\infty}\left(M_{1}\right.$, $\left.N_{1} \times N_{2}\right)$ be a map which is given by $\phi_{\sharp}(f)=(f, \phi)$. Then $\phi_{\#}$ is continuous.

(4) Let $\phi: M_{2} \rightarrow N_{2}$ be a smooth map and let $\phi_{!}: C^{\infty}\left(M_{1}, N_{1}\right) \rightarrow C^{\infty}\left(M_{1} \times\right.$ $\left.M_{2}, N_{1} \times N_{2}\right)$ be a map given by $\phi_{!}(f)=f \times \phi$. Then $\phi_{!}$is continuous.

(5) Let $\kappa: C^{\infty}\left(M_{1}, N_{1}\right) \times C^{\infty}\left(M_{1}, N_{2}\right) \rightarrow C^{\infty}\left(M_{1}, N_{1} \times N_{2}\right)$ be a map given by $\kappa(f, g)(x)=(f(x), g(x))$ for $x \in M_{1}$. Then $\kappa$ is continuous.

(6) Let $L$ be a smooth manifold. Let comp: $C^{\infty}\left(M_{1}, N_{1}\right) \times C^{\infty}\left(N_{1}, L\right) \rightarrow$ $C^{\infty}\left(M_{1}, L\right)$ be a map given by comp $(f, g)=g \circ f$. Then comp is continuous.

Proof. (1) and (2) are proved by R. Abraham [2, Theorems 11.2 and 11.3]. It is easy to see (3), (4) and (5). From J. Cerf [4, Chapter I, §4, Proposition 5], (6) follows.

Lemma 2.3. Let $X$ be a topological space. Let $M$ be a compact smooth manifold and $N$ be a smooth manifold. Choose an open covering $\left\{U_{i}\right\}$ of $M$ such that each closure $\bar{U}_{i}$ of $U_{i}$ is a regular submanifold of $M$ which is contained in a coordinate neighborhood of $M$. Then a map $\Psi: X \rightarrow C^{\infty}(M, N)$ is continuous if and only if each composition $\Psi_{i}: X \stackrel{\psi}{\longrightarrow} C^{\infty}(M, N) \stackrel{j_{i}^{*}}{\longrightarrow} C^{\infty}\left(\bar{U}_{i}, N\right)$ is continuous for each $i$, where $j_{i}: \bar{U}_{i} \hookrightarrow M$ is an inclusion.

Proof. From Lemma $2.2(2)$, if $\Psi$ is continuous, then $\Psi_{i}$ is continuous for each $i$. We can choose $\left\{U_{i}\right\}$ as a coordinate covering of $M$. Let $\left\{V_{\lambda}\right\}$ be a 
coordinate covering of $N$. Let $f \in C^{\infty}(M, N)$ and $K \subset U_{i}$ be a compact subset such that $f(K) \subset V_{\lambda}$ for some $\lambda$. $N^{r}\left(f, U_{i}, V_{\lambda}, K, \varepsilon\right)(r=0,1,2, \ldots, 0<\varepsilon \leq \infty)$ denotes the set of $C^{r}$ maps $g: M \rightarrow N$ such that $g(K) \subset V_{\lambda}$ and $\left\|D^{k} f(x)-D^{k} g(x)\right\|$ $<\varepsilon$ for any $x \in K, k=0,1,2, \ldots, r$. Then the $C^{\infty}$ topology on $C^{\infty}(M, N)$ is generated by these sets $N^{r}\left(f, U_{i}, V_{\lambda}, K, \varepsilon\right)$ (see M. Hirsch [6, Chapter 2, §1]).

Let $x \in X$ and let $f=\Psi(x)$. For any open neighborhood $W$ of $f$, there exist above sets $N_{k}=N^{r_{k}}\left(f, U_{i_{k}}, V_{\lambda_{k}}, K_{k}, \varepsilon_{k}\right), k=1,2, \ldots, n$, such that $\cap_{k=1}^{n} N_{k} \subset W$. Note that $j_{i_{k}}^{*}: C^{\infty}(M, N) \rightarrow C^{\infty}\left(\bar{U}_{i_{k}}, N\right)$ is an open map and $\left(j_{i_{k}}^{*}\right)^{-1}\left(j_{i_{k}}^{*}\left(N_{k}\right)\right)=N_{k}$. Since $\Psi_{i_{k}}$ is continuous, $\Psi^{-1}\left(N_{k}\right)=\Psi_{i_{k}}^{-1}\left(j_{i_{k}}^{*}\left(N_{k}\right)\right)$ is an open neighborhood of $x$ in $X$, for each $k$. Then $\cap_{k=1}^{n} \Psi^{-1}\left(N_{k}\right)$ is an open neighborhood of $x$ in $X$. Since $\Psi\left(\cap_{k=1}^{n} \Psi^{-1}\left(N_{k}\right)\right) \subset \cap_{k=1}^{n} N_{k} \subset W, \Psi$ is continuous at $x$. This completes the proof of Lemma 2.3.

Remark. Lemma 2.2 and Lemma 2.3 hold in the cases of manifolds with corners.

Let $C_{e}^{\infty}([-1 / 2,1 / 2], R)$ denote the set of all smooth functions $f:[-1 / 2$, $1 / 2] \rightarrow R$ satisfying $f(-x)=f(x)$, endowed with $C^{\infty}$ topology. Let $T$ : $C_{e}^{\infty}([-1 / 2,1 / 2], R) \rightarrow C^{\infty}([0,1 / 4], R)$ denote a map defined by $T(f)(x)$ $=f(\sqrt{x})$. Then we have

Lemma 2.4. The above map $T$ is well defined and continuous.

Proof. Put $f=T(\hat{f})$ for each $\hat{f} \in C_{e}^{\infty}([-1 / 2,1 / 2], R)$. Since $\hat{f}$ is a $C^{\infty}$ even function, we have the Taylor expansion

$$
\begin{aligned}
\hat{f}(x)=\hat{f}(0)+\left(\hat{f}^{\prime \prime}(0) / 2\right) x^{2}+\cdots & +\left(\hat{f}^{(2 n-2)}(0) /(2 n-2) !\right) x^{2 n-2} \\
+ & \left(\int_{0}^{1}\left((1-t)^{2 n-1} /(2 n-1) !\right) \hat{f}^{(2 n)}(t x) d t\right) x^{2 n}
\end{aligned}
$$

for $-1 / 2 \leq x \leq 1 / 2, n=1,2, \ldots$. Thus we have

$$
\begin{aligned}
f(x)=\hat{f}(0)+\left(\hat{f}^{\prime \prime}(0) / 2\right) x+\cdots & +\left(\hat{f}^{(2 n-2)}(0) /(2 n-2) !\right) x^{n-1} \\
+ & \left(\int_{0}^{1}\left((1-t)^{2 n-1} /(2 n-1) !\right) \hat{f}^{(2 n)}(t \sqrt{x}) d t\right) x^{n}
\end{aligned}
$$

for $0 \leq x \leq 1 / 4$. By the composite mapping formula, we can compute the $n$-th derivative

$$
\begin{aligned}
& D^{n}\left(\hat{f}^{(2 n)}(t \sqrt{x}) x^{n}\right) \\
& \quad=\sum_{p=0}^{n} \sum_{q=0}^{p} \sum_{\substack{i_{1}+\ldots+i_{q}=p \\
i_{1}>0, \ldots, i_{q}>0}} B\left(p, i_{1}, \ldots, i_{q}\right) \hat{f}^{(2 n+q)}(t \sqrt{x}) x^{q / 2} t^{q},
\end{aligned}
$$

where $B\left(p, i_{1}, \ldots, i_{q}\right)$ is a real number. Put $f_{i}=T\left(\hat{f}_{i}\right)$ for $\hat{f}_{i} \in C_{e}^{\infty}([-1 / 2,1 / 2], R)$ 
$(i=1,2)$. Then there exists a positive number $A_{n}$ such that

$$
\begin{aligned}
& \sup _{0 \leq x \leq 1 / 4}\left|D^{n} f_{1}(x)-D^{n} f_{2}(x)\right| \\
& \quad \leq A_{n} \cdot \max _{0 \leq q \leq 3 n}\left(\sup _{-1 / 2 \leq x \leq 1 / 2}\left|D^{q} \hat{f}_{1}(x)-D^{q} \hat{f}_{2}(x)\right|\right)
\end{aligned}
$$

for each $n=1,2, \ldots$. Note that

$$
\sup _{0 \leq x \leq 1 / 4}\left|f_{1}(x)-f_{2}(x)\right|=\sup _{-1 / 2 \leq x \leq 1 / 2}\left|\hat{f}_{1}(x)-\hat{f}_{2}(x)\right| \text {. }
$$

Therefore $T$ is a continuous map, and this completes the proof of Lemma 2.4.

Proof of Proposition 2.1. Let $J$ denote a closed interval $[0,1 / 4],[1 / 5$, $4 / 5]$ or $[3 / 4,1]$. By Lemma 2.3 , it is sufficient to show that the composition $P_{J}: \operatorname{Diff}_{G}^{\infty}(M)_{0} \stackrel{P}{\longrightarrow} \operatorname{Diff}^{\infty}[0,1] \stackrel{j *}{\longrightarrow} C^{\infty}(J,[0,1])$ is continuous, where $j: J \hookrightarrow$ $[0,1]$ is an inclusion map.

We shall first consider the case $J=[0,1 / 4]$. Let $\iota:[-1 / 2,1 / 2] \rightarrow[0,1 / 4]$ be a map given by $\iota(x)=x^{2}$. Let $\hat{\iota}:[-1 / 2,1 / 2] \rightarrow G \times{ }_{K_{0}} D\left(V_{0}\right) \hookrightarrow M$ be a map given by $\hat{\imath}(r)=\left[1, r e_{0}\right]$, where $e_{0}$ is a point of $S\left(V_{0}\right)$ as in $\S 1$. Then $\pi \circ \hat{\iota}=\iota$. Let $\hat{P}_{J}$ denote the composition $\operatorname{Diff}_{G}^{\infty}(M)_{0} \stackrel{\hat{\imath}^{*}}{\longrightarrow} C^{\infty}([-1 / 2,1 / 2], M) \stackrel{\pi_{*}}{\longrightarrow}$ $C^{\infty}([-1 / 2,1 / 2],[0,1])$. Then $\hat{P}_{J}(h)=\pi \circ h \circ \hat{\iota}=P(h) \circ \iota=\iota^{*} P(h)$ for $h \in$ $\operatorname{Diff}_{G}^{\infty}(M)_{0}$, and the image of $\hat{P}_{J}$ is contained in $C_{e}^{\infty}([-1 / 2,1 / 2], R)$. Note that $P_{J}=T \circ \hat{P}_{J}$. Combining Lemma 2.2 and Lemma 2.4, $P_{J}$ is continuous.

Next consider the case $J=[1 / 5,4 / 5]$. By Lemma 1.2, there is a $G$-diffeomorphism $\alpha: \pi^{-1}([1 / 5,4 / 5]) \rightarrow G / H \times[1 / 5,4 / 5]$. Let $i: \pi^{-1}([1 / 5,4 / 5]) \hookrightarrow M$ be the inclusion map and let $k:[1 / 5,4 / 5] \rightarrow G / H \times[1 / 5,4 / 5]$ be a map given by $k(r)=(1 H, r)$. Then $P_{J}$ is the composition

$$
\operatorname{Diff}_{G}^{\infty}(M)_{0} \stackrel{\left(i \circ \alpha^{-1 \circ k}\right) *}{\longrightarrow} C^{\infty}([1 / 5,4 / 5], M) \stackrel{\pi_{*}}{\longrightarrow} C^{\infty}([1 / 5,4 / 5],[0,1])
$$

which is continuous by Lemma 2.2.

We can prove that $P_{J}$ is continuous in the case $J=[3 / 4,1]$ similarly as in the case $J=[0,1 / 4]$, and this completes the proof of Proposition 2.1.

\section{§3. A Continuous Global Section of $\mathbb{P}$}

In Section 2 we have proved that $P: \operatorname{Diff}_{G}^{\infty}(M)_{0} \rightarrow \operatorname{Diff}^{\infty}[0,1]$ is continuous. Thus the image of $P$ is contained in the connected component $\operatorname{Diff}^{\infty}[0,1]_{0}$ of the identity. In this section we shall construct a continuous global section of $P: \operatorname{Diff}_{G}^{\infty}(M)_{0} \rightarrow \operatorname{Diff}^{\infty}[0,1]_{0}$.

Let $f$ be an element of $\operatorname{Diff}^{\infty}[0,1]_{0}$. We shall define a map $\Psi(f): M \rightarrow M$ as follows: $\Psi(f)$ is defined on $\pi^{-1}((0,1))$ by the composition $\pi^{-1}((0,1)) \stackrel{\alpha}{\longrightarrow}$ 
$G / H \times(0,1) \stackrel{(1, f)}{\longrightarrow} G / H \times(0,1) \stackrel{\alpha^{-1}}{\longrightarrow} \pi^{-1}((0,1))$, and $\Psi(f)=1$ on $\pi^{-1}(0) \cup \pi^{-1}(1)$.

Proposition 3.1. $\Psi(f)$ is a G-diffeomorphism of $M$.

In order to prove Proposition 3.1, we need the following lemma and notations.

Lemma 3.2. Let $\Psi_{1}: \operatorname{Diff}^{\infty}[0,1]_{0} \rightarrow \operatorname{Diff}^{\infty}\left(D^{n}\right)$ be a map defined by

$$
\Psi_{1}(f)(v)=\left\{\begin{array}{ccc}
\left.\left(\sqrt{f\left(\|v\|^{2}\right.}\right) /\|v\|\right) v & \text { for } & v \neq 0, \\
0 & \text { for } & v=0,
\end{array}\right.
$$

where $D^{n}$ denotes an $n$-dimensional unit disc. Then $\Psi_{1}$ is well defined and continuous.

Notations 3.3. For $i=0,1$, we shall $u$ se the following notations

$\pi_{i}: G \rightarrow G / K_{i}$ the natural projection,

$U_{i}$ an open disc neighborhood of $1 K_{i}$ in $G / K_{i}$,

$\sigma_{i}: U_{i} \rightarrow G \quad$ a smooth local cross section of $\pi_{i}$,

$\sigma_{i, a}: a U_{i} \rightarrow G(a \in G) \quad a$ smooth local cross section of $\pi_{i}$ defined by $\sigma_{i, a}(x)=a \cdot \sigma_{i}\left(a^{-1} x\right)$.

Put $M_{i}=G \times{ }_{K_{i}} D\left(V_{i}\right)$ and $M_{i}(r)=G \times{ }_{K_{i}} D_{r}\left(V_{i}\right)$, where $D_{r}\left(V_{i}\right)$ is a closed $r$-disc in $V_{i}(0<r \leq 1)$.

$p_{i}: M_{i} \rightarrow G / K_{i}, p_{i, r}: M_{i}(r) \rightarrow G / K_{i}$ the bundle projections,

$\phi_{i, a}: p_{i}^{-1}\left(a U_{i}\right) \rightarrow U_{i} \times D\left(V_{i}\right)(a \in G)$ a chart of $p_{i}$ over $a U_{i}$ defined $b y$ $\phi_{i, a}([g, v])=\left(a^{-1} \pi_{i}(g),\left(\left(\sigma_{i, a} \circ \pi_{i}\right)(g)\right)^{-1} g \cdot v\right)$,

$\pi_{2}: G \rightarrow G / H$ the natural projection,

$U_{2}$ an open disc neighborhood of $1 H$ in $G / H$,

$\sigma_{2}: U_{2} \rightarrow G$ a smooth local cross section of $\pi_{2}$.

Proof of Proposition 3.1. Put $h=\Psi(f)$. We shall first prove that $h$ is smooth on $\pi^{-1}(0)$. Since $f(0)=0$, there exists a real number $\varepsilon$ such that $0<\varepsilon \leq$ $1 / 2$ and $f\left(\varepsilon^{2}\right) \leq 1 / 4$. Then $h\left(\pi^{-1}\left(\left[0, \varepsilon^{2}\right]\right)\right) \subset \pi^{-1}([0,1 / 4])$, and $h\left(M_{0}(\varepsilon)\right) \subset$ $M_{0}(1 / 2)$. For $\left[g, r e_{0}\right] \in G \times_{K_{0}} D_{\varepsilon}\left(V_{0}-0\right)(0<r \leq \varepsilon), h\left(\left[g, r e_{0}\right]\right)=\left(\alpha^{-1} \circ(1, f) \circ \alpha\right)$ $\left(\left[g, r e_{0}\right]\right)=\left(\alpha^{-1} \circ(1, f)\right)\left(g H, r^{2}\right)=\alpha^{-1}\left(g H, f\left(r^{2}\right)\right)=\left[g, \sqrt{f\left(r^{2}\right)} e_{0}\right]$. Then, for $\left.[g, v] \in G \times_{K_{0}} D_{\varepsilon}\left(V_{0}-0\right), h([g, v])=\left[g, \sqrt{f\left(\|v\|^{2}\right.}\right) /\|v\| v\right]=\left[g, \Psi_{1}(f)(v)\right]$. Since $h([g, 0])=[g, 0], h([g, v])=\left[g, \Psi_{1}(f)(v)\right]$ for any $[g, v] \in M_{0}(\varepsilon)$. Then the composition

$$
\begin{aligned}
\tilde{h}: U_{0} \times D_{\varepsilon}\left(V_{0}\right) & \stackrel{\left(\phi_{0, a}\right)^{-1}}{\longrightarrow} p_{0, \varepsilon}^{-1}\left(a U_{0}\right) \\
& \stackrel{h}{\longrightarrow} p_{0,1 / 2}^{-1}\left(a U_{0}\right) \\
\stackrel{\phi_{0, a}}{\longrightarrow} & U_{0} \times D_{1 / 2}\left(V_{0}\right)
\end{aligned}
$$


is given by $\tilde{h}(x, v)=\left(x, \Psi_{1}(f)(v)\right)$ for $a \in G$. Since $\Psi_{1}(f)$ is a smooth map by Lemma $3.2, h$ is smooth on $\pi^{-1}(0)$. Similarly we can prove that $h$ is smooth on $\pi^{-1}(1)$. Since $h$ is smooth on $\pi^{-1}((0,1))$ by the definition, $h$ is a smooth map. Since $h^{-1}=\Psi\left(f^{-1}\right), h^{-1}$ is also a smooth map. Thus $h$ is a $G$-diffeomorphism of $M$, and this completes the proof of Proposition 3.1.

In order to prove Lemma 3.2, we need the following assertion.

Assertion 3.4. Let $\Phi: \operatorname{Diff}^{\infty}[0,1]_{0} \rightarrow C^{\infty}([0,1], R)$ be a map given by

$$
\Phi(f)(x)= \begin{cases}\sqrt{f(x) / x} & \text { for } x \neq 0, \\ \sqrt{f^{\prime}(0)} & \text { for } x=0 .\end{cases}
$$

Then $\Phi$ is well defined and continuous.

Proof. For $f \in \operatorname{Diff}^{\infty}[0,1]_{0}$, we have the Taylor expansion

$$
f(x)=f^{\prime}(0) x+x^{2} \int_{0}^{1}(1-t) f^{\prime \prime}(t x) d t \quad \text { for } \quad 0 \leq x \leq 1 .
$$

Put $F(x)=f^{\prime}(0)+x \int_{0}^{1}(1-t) f^{\prime \prime}(t x) d t$ for $0 \leq x \leq 1$. Then $\Phi(f)=\sqrt{F}$. Note that $F(x)>0$ for $0 \leq x \leq 1$. It is easy to see that $\Phi$ is continuous.

Proof of Lemma 3.2. Let $N: D^{n} \rightarrow[0,1]$ be a map given by $N(v)=\|v\|^{2}$. Let $i: D^{n} \hookrightarrow R^{n}$ be the inclusion and let $\mu: R \times R^{n} \rightarrow R^{n}$ be the scalar multiplication. Since $\Psi_{1}(f)(v)=\Phi(f)\left(\|v\|^{2}\right) v, \Psi_{1}(f)$ is a smooth map by Assertion 3.4. Since $\Psi_{1}\left(f^{-1}\right)=\Psi_{1}(f)^{-1}, \Psi_{1}(f)^{-1}$ is also a smooth map. Thus $\Psi_{1}(f)$ is a diffeomorphism of $D^{n}$. Note that $\Psi_{1}$ is the composition $\operatorname{Diff}^{\infty}[0,1]_{0} \stackrel{\oplus}{\longrightarrow}$ $C^{\infty}([0,1], R) \stackrel{N^{*}}{\longrightarrow} C^{\infty}\left(D^{n}, R\right) \stackrel{i_{\#}}{\longrightarrow} C^{\infty}\left(D^{n}, R \times R^{n}\right) \stackrel{\mu_{*}}{\longrightarrow} C^{\infty}\left(D^{n}, R^{n}\right)$. Combining Assertion 3.4 and Lemma 2.2, $\Psi_{1}$ is continuous. This completes the proof of Lemma 3.2.

Proposition 3.5. $\Psi: \operatorname{Diff}^{\infty}[0,1]_{0} \rightarrow \operatorname{Diff}_{G}^{\infty}(M)$ is continuous.

Proof. Let $B_{i} \subset U_{i}$ be a closed disc neighborhood of $1 K_{i}$ in $G / K_{i}$ for $i=0,1$. Let $B_{2} \subset U_{2}$ be a closed disc neighborhood of $1 H$ in $G / H$. We can choose $\left\{\operatorname{int}\left(p_{0, \varepsilon}^{-1}\left(a B_{0}\right)\right)\right.$, int $\left(p_{1, \varepsilon}^{-1}\left(a B_{1}\right)\right)$, int $\left.\left(\alpha^{-1}\left(a B_{2} \times[\varepsilon / 2,1-\varepsilon / 2]\right)\right) ; a \in G\right\}$ as an open covering of $M$ for $0<\varepsilon<1 / 2$. Put $W=\left\{f \in \operatorname{Diff}^{\infty}[0,1]_{0} ; f\left(\left[0, \varepsilon^{2}\right]\right) \subset\right.$ $\left.[0,1 / 4), f\left(\left[1-\varepsilon^{2}, 1\right]\right) \subset(3 / 4,1]\right\}$. Then $W$ is an open neighborhood of the identity in $\operatorname{Diff}^{\infty}[0,1]_{0}$. Since $\Psi$ is a homomorphism as an abstract group, it is enough to show that $\Psi$ is continuous on $W$. Let $C$ denote one of the sets $p_{0, \varepsilon}^{-1}\left(a B_{0}\right), p_{1, \varepsilon}^{-1}\left(a B_{1}\right)$ or $\alpha^{-1}\left(a B_{2} \times[\varepsilon / 2,1-\varepsilon / 2]\right)$ for $a \in G$. If we can prove that the composition 


$$
\Psi_{C}: W \stackrel{\Psi}{\longrightarrow} \operatorname{Diff}_{G}^{\infty}(M)_{0} \stackrel{i^{*}}{\longrightarrow} C^{\infty}(C, M)
$$

is continuous for each $C$, then $\Psi$ is continuous on $W$ by Lemma 2.3, where $i: C \hookrightarrow M$ is an inclusion map.

First consider in the case $C=p_{0, \varepsilon}^{-1}\left(a B_{0}\right) . \quad \Psi(f)(C)$ is contained in $p_{0,1 / 2}^{-1}\left(a U_{0}\right)$ for each $f \in W$. Note that $\Psi(f)([g, v])=\left[g, \Psi_{1}(f)(v)\right]$ for $[g, v] \in C$ and $\left(\phi_{0, a} \circ \Psi(f) \circ \phi_{0, a}^{-1}\right)(x, v)=\left(x, \Psi_{1}(f)(v)\right)$ for $(x, v) \in B_{0} \times D_{\varepsilon}\left(V_{0}\right)$. Thus $\Psi_{C}$ is given by the composition

$$
\begin{aligned}
W & \stackrel{\Psi_{1}}{\longrightarrow} C^{\infty}\left(D_{\varepsilon}\left(V_{0}\right), D\left(V_{0}\right)\right) \\
\stackrel{j_{!}}{\longrightarrow} & C^{\infty}\left(B_{0} \times D_{\varepsilon}\left(V_{0}\right), U_{0} \times D\left(V_{0}\right)\right) \\
& \stackrel{\phi_{0, a^{*}}}{\longrightarrow} C^{\infty}\left(C, U_{0} \times D\left(V_{0}\right)\right) \\
\stackrel{\left(k^{\circ} \phi_{0, a}^{-1}\right) *}{\longrightarrow} & C^{\infty}(C, M),
\end{aligned}
$$

where $j: B_{0} \hookrightarrow U_{0}$ and $k: p_{0}^{-1}\left(a U_{0}\right) \hookrightarrow M$ are inclusions. Combining Lemma 3.2 and Lemma 2.2, $\Psi_{C}$ is continuous.

Now consider the case $C=\alpha^{-1}\left(B_{0} \times[\varepsilon / 2,1-\varepsilon / 2]\right) . \quad \Psi_{C}$ is given by the composition

$$
\begin{aligned}
& W \stackrel{\iota^{*}}{\longrightarrow} C^{\infty}([\varepsilon / 2,1-\varepsilon / 2],(0,1)) \\
& \stackrel{j_{!}}{\longrightarrow} C^{\infty}\left(B_{0} \times[\varepsilon / 2,1-\varepsilon / 2], G / H \times(0,1)\right) \\
& \stackrel{\alpha^{*}}{\longrightarrow} C^{\infty}(C, G / H \times(0,1)) \\
& \stackrel{\left(k \circ \alpha^{-1}\right)_{*}}{\longrightarrow} C^{\infty}(C, M) \text {, }
\end{aligned}
$$

where $\iota:[\varepsilon / 2,1-\varepsilon / 2] \hookrightarrow[0,1], j: B_{0} \hookrightarrow G / H$ and $k: \pi^{-1}((0,1)) \hookrightarrow M$ are inclusion maps. By Lemma 2.2, $\Psi_{C}$ is continuous.

We can prove that $\Psi_{C}$ is continuous in the case $C=p_{1, \varepsilon}^{-1}\left(a B_{1}\right)$ similarly as in the case $C=p_{0, \varepsilon}^{-1}\left(a B_{0}\right)$, and this completes the proof of Proposition 3.5.

By Proposition 3.5, $P: \operatorname{Diff}_{G}^{\infty}(M)_{0} \rightarrow \operatorname{Diff}^{\infty}[0,1]_{0}$ is a globally trivial fibration. Then we have

Corollary 3.6. $\operatorname{Diff}_{G}^{\infty}(M)_{0}$ is homeomorphic to $\operatorname{Diff}^{\infty}[0,1]_{0} \times \operatorname{Ker} P$.

\section{§4. On the Group Ker $P$}

In this section we shall define a group homomorphism $L: \operatorname{Ker} P \rightarrow Q$, where $Q$ is a subgroup of $C^{\infty}([0,1], N(H) / H)$, and we shall prove that $L$ is a group monomorphism between topological groups (see Lemma 4.5 and Proposition 4.6). 
Let $h$ be an element of $\operatorname{Ker} P$. Let $\hat{h}$ be the composition

$$
G / H \times(0,1) \stackrel{\alpha^{-1}}{\longrightarrow} \pi^{-1}((0,1)) \stackrel{h}{\longrightarrow} \pi^{-1}((0,1)) \stackrel{\alpha}{\longrightarrow} G / H \times(0,1) .
$$

Then $\hat{h}$ is a level preserving $G$-diffeomorphism. Let $a:(0,1) \rightarrow N(H) / H$ be a smooth map satisfying $h(g H, r)=(g a(r), r)$ for $(g H, r) \in G / H \times(0,1)$.

Proposition 4.1. With the above notations, there exists a smooth map $\bar{a}:[0,1] \rightarrow N(H) / H$ such that

(1) $\bar{a}=a$ on $(0,1)$,

(2) $\bar{a}(i) \in\left(N(H) \cap N\left(K_{i}\right)\right) / H$ for $i=0,1$.

To prove Proposition 4.1, we need the following lemmas.

Lemma 4.2. Let $G$ be a compact Lie group. Let $K$ and $N$ be closed subgroups of $G$. Let $\pi: G \rightarrow G / K$ be the natural projection. Then there exists a smooth local section $\sigma$ of $\pi$, which is defined on an open neighborhood $U$ of $1 K$, such that $\sigma(1 K)=1$ and $\sigma(x) \in N$ for $x \in \pi(N) \cap U$.

Proof. Let $\pi_{1}: N \rightarrow N /(N \cap K)$ be a natural projection. Let $i: N \hookrightarrow G$ be the inclusion and let $I: N /(N \cap K) \rightarrow G / K$ be a map satisfying $\pi \circ i=I \circ \pi_{1}$. Since $I(N /(N \cap K))=\pi(N)$ is an orbit of the natural action $N \times G / K \rightarrow G / K, I$ is an imbedding. Let $U$ be a disc neighborhood around $\pi(1)$ in $G / K$ and let $U_{1}$ be a disc neighborhood around $\pi_{1}(1)$ in $N /(N \cap K)$. Since $I$ is an imbedding, we can assume $I\left(U_{1}\right)=U \cap I(N /(N \cap K))=U \cap \pi(N)$. Let $\sigma_{1}: U_{1} \rightarrow N$ be a smooth local section of $\pi_{1}$ satisfying $\sigma_{1}(\pi(1))=1$. Then $\sigma_{1} \circ I^{-1}$ is a smooth section defined on $I\left(U_{1}\right)$. We can extend $\sigma_{1} \circ I^{-1}$ to a smooth local section defined on $U$. Then $\sigma(\pi(1))=1$ and $\sigma(U \cap \pi(N)) \subset N$. This completes the proof of Lemma 4.2.

Lemma 4.3. Let $G$ be a compact connected Lie group. Let $V$ be a representation of $G$ such that $G$ acts transitively and effectively on a unit sphere $S(V)$ of $V$. Let $H$ be the isotropy subgroup of a point of $S(V)$. Then we have the following list:

\begin{tabular}{|c|c|c|c|c|c|}
\hline$G$ & $S O(n) \quad(n \geq 3)$ & $S U(n) \quad(n \geq 2)$ & $U(n) \quad(n \geq 1)$ & $S p(n) \quad(n \geq 1)$ & $S p(n) \times_{Z_{2}} S^{3} \quad(n \geq 1)$ \\
\hline$H$ & $S O(n-1)$ & $S U(n-1)$ & $U(n-1)$ & $S p(n-1)$ & $H_{1}$ \\
\hline$N(H) / H$ & $Z_{2}$ & $U(1)$ & $U(1)$ & $S p(1)$ & $Z_{2}$ \\
\hline
\end{tabular}




\begin{tabular}{|c|c|c|c|}
\hline$S p(n) \times_{z_{2}} S^{1} \quad(n \geq 1)$ & $G_{2}$ & $\operatorname{Spin}(7)$ & $\operatorname{Spin}(9)$ \\
\hline$H_{2}$ & $S U(3)$ & $G_{2}$ & $\operatorname{Spin}(7)$ \\
\hline$S^{1}$ & $Z_{2}$ & $Z_{2}$ & $Z_{2}$ \\
\hline
\end{tabular}

where $H_{1}=\left\{\left[(q, A), q^{-1}\right] \in S p(n) \times{ }_{Z_{2}} S^{3} ;(q, A) \in S p(1) \times S p(n-1) \subset S p(n)\right\}$ and $H_{2}=\left\{\left[(z, A), z^{-1}\right] \in S p(n) \times{ }_{z_{2}} S^{1} ;(z, A) \in S^{1} \times S p(n-1) \subset S p(n)\right\}$.

Proof. It is known that $G$ and $H$ are the above Lie groups (c.f. W. C. Hsiang and W. Y. Hsiang $[7, \S 1])$. We can determine the Lie group $N(H) / H$ by an immediate calculation except for $G=G_{2}$, Spin (7), Spin (9). For the cases $G=G_{2}$, Spin (7), Spin (9), we can determine $N(H) / H$ by using I. Yokota's definitions of these Lie groups in [9, Chapter 5].

Lemma 4.4. (1) Let $F:[-1,1] \rightarrow R$ be a smooth function such that $F(0)=0$. Put $f(x)=F(x) / x$ for $x \neq 0$ and $f(x)=F^{\prime}(0)$ for $x=0$. Then $f:[-1,1]$ $\rightarrow R$ is a well defined smooth function.

(2) Put $C_{0}^{\infty}([-1,1], R)=\left\{F \in C^{\infty}([-1,1], R) ; F(0)=0\right\}$, endowed with $C^{\infty}$ topology. Let $\Phi: C_{0}^{\infty}([-1,1], R) \rightarrow C^{\infty}([-1,1], R)$ be a map given by $\Phi(F)(x)=f(x)$. Then $\Phi$ is continuous.

Proof. For $F \in C_{0}^{\infty}([-1,1], R)$, we have $\Phi(F)(x)=f(x)=F^{\prime}(0)$ $+x \int_{0}^{1}(1-t) F^{\prime \prime}(t x) d t$. Then the $n$-th derivative $f^{(n)}(x)=x \int_{0}^{1}(1-t) t^{n} F^{(n+2)}(t x) d t$ $+n \int_{0}^{1}(1-t) t^{n-1} F^{(n+1)}(t x) d t$. Thus there exists a positive number $A$ such that $\|\Phi(F)\|_{n} \leq A\|F\|_{n+2}$, and Lemma 4.4 follows.

Proof of Proposition 4.1. Let $\varepsilon(0<\varepsilon \leq 1 / 2)$ be a real number. Let $W_{i}$ and $U_{i}$ be open neighborhoods of $1 K_{i}$, satisfying $\bar{W}_{i} \subset U_{i}$, for $i=0,1$. Put $O=\left\{h \in \operatorname{Ker} P ; h\left(p_{i, \varepsilon}^{-1}\left(\overline{W_{i}}\right)\right) \subset p_{i, \varepsilon}^{-1}\left(U_{i}\right)\right.$ for $\left.i=0,1\right\}$. Then $O$ is an open neighborhood of the identity in $\operatorname{Ker} P$. By Corollary 3.6, Ker $P$ is connected, and $O$ generates the topological group $\operatorname{Ker} P$. Thus we can assume $h \in O$.

Let $\tilde{h}$ be the composition

$$
W_{0} \times D_{\varepsilon}\left(V_{0}\right) \stackrel{\left(\phi_{0,1}\right)^{-1}}{\longrightarrow} p_{0, \varepsilon}^{-1}\left(W_{0}\right) \stackrel{h}{\longrightarrow} p_{0, \varepsilon}^{-1}\left(U_{0}\right) \stackrel{\phi_{0,1}}{\longrightarrow} U_{0} \times D_{\varepsilon}\left(V_{0}\right) .
$$

Let $\rho_{1}: U_{0} \times D_{\varepsilon}\left(V_{0}\right) \rightarrow U_{0}$ and $\rho_{2}: U_{-0} \times D_{\varepsilon}\left(V_{0}\right) \rightarrow D_{\varepsilon}\left(V_{0}\right)$ be projections on the first factor and the second factor, respectively. Let $i:[-\varepsilon, \varepsilon] \rightarrow W_{0} \times D_{\varepsilon}\left(V_{0}\right)$ be an imbedding given by $i(r)=\left(1 K_{0}, r e_{0}\right)$. Then the compositions $\tilde{h}_{1}=\rho_{1} \circ \tilde{h} \circ i$ : $[-\varepsilon, \varepsilon] \rightarrow U_{0}$ and $\tilde{h}_{2}=\rho_{2} \circ \tilde{h} \circ i:[-\varepsilon, \varepsilon] \rightarrow D_{\varepsilon}\left(V_{0}\right)$ are smooth maps. Let $\bar{\pi}_{0}: G / H$ $\rightarrow G / K_{0}$ be the natural projection. Note that 


$$
\begin{aligned}
\left(\alpha \circ h \circ \phi_{0,1}^{-1}\right)\left(1 K_{0}, r e_{0}\right) & =(\alpha \circ h)\left(\left[1, r e_{0}\right]\right) \\
& =(\hat{h} \circ \alpha)\left(\left[1, r e_{0}\right]\right) \\
& =\hat{h}\left(1 H, r^{2}\right) \\
& =\left(a\left(r^{2}\right), r^{2}\right) \quad \text { for }|r| \leq \varepsilon, r \neq 0 .
\end{aligned}
$$

Then

$$
\begin{aligned}
\tilde{h}\left(1 K_{0}, r e_{0}\right) & =\left(\phi_{0,1} \circ \alpha^{-1}\right)\left(a\left(r^{2}\right), r^{2}\right) \\
& =\left(\bar{\pi}_{0}\left(a\left(r^{2}\right)\right), \quad\left(\sigma_{0} \circ \bar{\pi}_{0}\right)\left(a\left(r^{2}\right)\right)^{-1} \cdot a\left(r^{2}\right) \cdot r e_{0}\right),
\end{aligned}
$$

and

$$
\begin{aligned}
& \tilde{h}_{1}(r)=\bar{\pi}_{0}\left(a\left(r^{2}\right)\right), \\
& \tilde{h}_{2}(r)=\left(\sigma_{0} \circ \bar{\pi}_{0}\right)\left(a\left(r^{2}\right)\right)^{-1} \cdot a\left(r^{2}\right) \cdot r e_{0},
\end{aligned}
$$

for $|r| \leq \varepsilon, r \neq 0$.

Here we can assume that $\sigma_{0}\left(1 K_{0}\right)=1$ and $\sigma_{0}\left(\pi_{0}(N(H)) \cap U_{0}\right) \subset N(H)$ by Lemma 4.2. Let $b:[-\varepsilon, \varepsilon] \rightarrow G$ be a smooth map given by $b(r)=\sigma_{0}\left(\tilde{h}_{1}(r)\right)$. Then $b(r)=\sigma_{0}\left(\bar{\pi}_{0}\left(a\left(r^{2}\right)\right)\right) \in \sigma_{0}\left(\pi_{0}(N(H)) \cap U_{0}\right)$, and $b(r) \in N(H)$ for $r \neq 0$. Since $b$ is a smooth map, $b(r) \in N(H)$ for $r=0$. For $[1,0] \in \pi^{-1}(0)$, we have $h([1,0])$ $=\left(h \circ \phi_{0,1}^{-1}\right)\left(1 K_{0}, 0\right)=\left(h \circ \phi_{0,1}^{-1}\right)(i(0))=\phi_{0,1}^{-1}\left(\tilde{h}_{1}(0), 0\right)=[b(0), 0]$. Note that $p_{0}$ is a $G$-diffeomorphism on the zero section of $p_{0}$ and $h\left(\pi^{-1}(0)\right)=\pi^{-1}(0)$. Then the composition $p_{0} \circ h \circ p_{0}^{-1}: G / K_{0} \rightarrow G / K_{0}$ is a $G$-diffeomorphism, and $\left(p_{0} \circ h \circ p_{0}^{-1}\right)$ $\left(1 K_{0}\right)=\left(p_{0} \circ h\right)([1,0])=p_{0}([b(0), 0])=b(0) K_{0}$. Thus $b(0) \in N\left(K_{0}\right)$, and $b(0) \in$ $N(H) \cap N\left(K_{0}\right)$.

Put $J=[-\varepsilon, 0) \cup(0, \varepsilon]$. Let $c: J \rightarrow N(H) / H$ be a smooth map given by $c(r)=b(r)^{-1} \cdot a\left(r^{2}\right)$. Since $\bar{\pi}_{0}(c(r))=\bar{\pi}_{0}\left(\sigma_{0}\left(\bar{\pi}_{0}\left(a\left(r^{2}\right)\right)\right)^{-1} \cdot a\left(r^{2}\right)\right)=1 K_{0}$, then $c(r)$ $\in K_{0} / H$. Thus $c(r) \in N\left(H, K_{0}\right) / H$ for $r \in J$. Since Ker $P$ is connected, the maps $a, b$ and $c$ are homotopic to the constant maps. Note that the identity component $\left(N\left(H, K_{0}\right) / H\right)^{0}$ of $N\left(H, K_{0}\right) / H$ is contained in $\left(N\left(H, K_{0}\right) \cap K_{0}^{0}\right) \cdot H / H$, and there exists an isomorphism $\left(N\left(H, K_{0}\right) \cap K_{0}^{0}\right) \cdot H / H \simeq\left(N\left(H, K_{0}\right) \cap K_{0}^{0}\right) /$ $\left(H \cap K_{0}^{0}\right)$ as a Lie group, where $K_{0}^{0}$ is the identity component of $K_{0}$. Then there exists a smooth map $\hat{c}: J \stackrel{c}{\longrightarrow}\left(N\left(H, K_{0}\right) / H\right)^{\circ \hookrightarrow}\left(N\left(H, K_{0}\right) \cap K_{0}^{0}\right) \cdot H / H \simeq(N(H$, $\left.\left.K_{0}\right) \cap K_{0}^{0}\right) /\left(H \cap K_{0}^{0}\right) \hookrightarrow N\left(H \cap K_{0}^{0}, K_{0}^{0}\right) /\left(H \cap K_{0}^{0}\right)$. Now we shall prove that $\hat{c}$ can be extended to a smooth map on $[-\varepsilon, \varepsilon]$, and so is $c$.

Note that $K_{0}$ acts transitively on the unit sphere $S\left(V_{0}\right)$ of $V_{0}$. If $\operatorname{dim} S\left(V_{0}\right)$ $=0$, then $K_{0} / H=Z_{2}$ and $N\left(H, K_{0}\right) / H=Z_{2}$. In this case $\hat{c}$ is a trivial map, and it is clear that $\hat{c}$ can be extended to a smooth map on $[-\varepsilon, \varepsilon]$. Now we assume $\operatorname{dim} S\left(V_{0}\right)>0$. Since $S\left(V_{0}\right)$ is connected, $K_{0}^{0}$ acts transitively on $S\left(V_{0}\right)$ and $K_{0}^{0} /\left(K_{0}^{0} \cap H\right)$ is diffeomorphic to $S\left(V_{0}\right)$. Put $D=\cap_{g \in K_{0}^{0}} g\left(K_{0}^{0} \cap H\right) g^{-1}$ which is 
the kernel of the action $K_{0}^{0} \times S\left(V_{0}\right) \rightarrow S\left(V_{0}\right)$. Put $\bar{K}_{0}=K_{0}^{0} / D$ and $\bar{H}=\left(H \cap K_{0}^{0}\right) /$ D. Then $\bar{K}_{0}$ acts transitively and effectively on $S\left(V_{0}\right)$ and $\bar{K}_{0} / \bar{H}$ is diffeomorphic to $S\left(V_{0}\right)$. Put $\bar{N}_{0}=N\left(\bar{H}, \bar{K}_{0}\right) / \bar{H}$ which is isomorphic to $N\left(H \cap K_{0}^{0}, K_{0}^{0}\right) /$ $\left(H \cap K_{0}^{0}\right)$ as a Lie group. The pair $\left(\bar{K}_{0}, \bar{N}_{0}\right)$ is one of pairs $(G, N(H) / H)$ in the list of Lemma 4.3. Now we shall prove that $\hat{c}$ can be extended to a smooth map on $[-\varepsilon, \varepsilon]$. If $\bar{N}_{0}=Z_{2}$, this is clear since $\hat{c}$ is a trivial map.

Consider the case $\bar{K}_{0}=S U(n)(n \geq 1)$ and $\bar{N}_{0}=U(1)$. In this case $V_{0}$ is an $n$-dimensional complex vector space and $\bar{N}_{0}=U(1)$ acts on $V_{0}$ as a scalar multiplication. We can regard $C^{n}$ as a $2 n$-dimensional real vector space $R^{2 n}$ and $\bar{N}_{0}$ as $S O(2)$. Then there exist smooth functions $c_{i}: J \rightarrow R, i=1,2$, such that

$$
\hat{c}(r)=\left[\begin{array}{cc}
c_{1}(r) & -c_{2}(r) \\
c_{2}(r) & c_{1}(r)
\end{array}\right] \in S O(2) \quad \text { for } \quad r \in J .
$$

Note that $\tilde{h}_{2}:[-\varepsilon, \varepsilon] \rightarrow D_{\varepsilon}\left(V_{0}\right)$ is a smooth map and $\tilde{h}_{2}(r)=c(r) \cdot r e_{0}=\hat{c}(r) \cdot r e_{0}$ for $r \neq 0$. In this case $e_{0}=(1,0, \ldots, 0) \in S^{2 n-1}$ and $\tilde{h}_{2}(r)=\left(c_{1}(r) r, c_{2}(r) r, 0, \ldots, 0\right)$ for $r \in J$. Put $c_{i}(0)=\lim _{r \rightarrow 0} c_{i}(r)$ for $i=1,2$. From Lemma 4.4, $c_{i}:[-\varepsilon, \varepsilon] \rightarrow$ $R, i=1,2$, are smooth functions and $\hat{c}$ can be extended to a smooth map on $[-\varepsilon, \varepsilon]$.

Now consider the case $\bar{K}_{0}=S p(n)(n \geq 1)$ and $\bar{N}=S p(1)$. In this case $V_{0}$ is an $n$-dimensional quaternionic vector space $H^{n}$ and $\bar{N}_{0}=S p(1)$ acts on $V_{0}$ as a scalar multiplication. We can regard $H^{n}$ as $R^{4 n}$ and $S p(1)$ as a subgroup of $S O(4)$ naturally. By the similar way as in the case $K_{0}=S U(n)$, there exist smooth functions $c_{i}: J \rightarrow R, i=1,2,3,4$, such that $h_{2}(r)=\left(c_{1}(r) r, c_{2}(r) r, c_{3}(r) r, c_{4}(r) r\right.$, $0, \ldots, 0)$ for $r \in J$, and we can extend $\hat{c}$ to a smooth map on $[-\varepsilon, \varepsilon]$.

The proofs of the other cases are similar to those of the above cases. Thus we can extend $c$ to a smooth map on $[-\varepsilon, \varepsilon]$. Since $c(r) \in N\left(H, K_{0}\right) / H$ for $r \neq 0$, we see $c(0) \in N\left(H, K_{0}\right) / H$. Put $\bar{a}(0)=b(0) \cdot c(0)$. Since $b(0) \in N(H) \cap$ $N\left(K_{0}\right)$ and $c(0) \in N\left(H, K_{0}\right) / H$, we have $\bar{a}(0) \in\left(N(H) \cap N\left(K_{0}\right)\right) / H$. Let $\hat{a}$ : $[-1 / 2,1 / 2] \rightarrow N(H) / H$ be a map given by $\hat{a}(r)=\bar{a}\left(r^{2}\right)$. Since $\hat{a}(r)=b(r) \cdot c(r)$ for $-\varepsilon \leq r \leq \varepsilon, \hat{a}$ is a smooth map. Since $\hat{a}$ is an even map and $\bar{a}(r)=\hat{a}(\sqrt{r})$ for $0 \leq r \leq 1 / 4, \bar{a}$ is a smooth map on $[0,1 / 4]$ by Lemma 2.4. Thus we can extend the map $a$ to a smooth map $\bar{a}$ on $[0,1)$ satisfying $\bar{a}(0) \in\left(N(H) \cap N\left(K_{0}\right)\right) / H$. Similarly we can extend $a$ to a smooth map $\bar{a}$ on $[0,1]$ satisfying $\bar{a}(1) \in(N(H) \cap$ $\left.N\left(K_{1}\right)\right) / H$. This completes the proof of Proposition 4.1.

Let $Q$ denote the set of smooth maps $f:[0,1] \rightarrow N(H) / H$ satisfying $f(i) \in$ $\left(N(H) \cap N\left(K_{i}\right)\right) / H$ for $i=0,1$, endowed with $C^{\infty}$ topology. Using Proposition 
4.1, we define a map $L: \operatorname{Ker} P \rightarrow Q$ by $L(h)=\bar{a}^{-1}$.

Lemma 4.5. L: Ker $P \rightarrow Q$ is a group monomorphism.

Proof. Let $h_{i} \in \operatorname{Ker} P$ for $i=1,2$. For $0<r<1$ and $g \in G$, we have

$$
\begin{aligned}
\left(g \cdot L\left(h_{2} \circ h_{1}\right)(r)^{-1}, r\right) & =\left(\alpha \circ h_{2} \circ h_{1} \circ \alpha^{-1}\right)(g H, r) \\
& =\left(\left(\alpha \circ h_{2} \circ \alpha^{-1}\right) \circ\left(\alpha \circ h_{1} \circ \alpha^{-1}\right)\right)(g H, r) \\
& =\left(\alpha \circ h_{2} \circ \alpha^{-1}\right)\left(g \cdot L\left(h_{1}\right)(r)^{-1}, r\right) \\
& =\left(g \cdot L\left(h_{1}\right)(r)^{-1} \cdot L\left(h_{2}\right)(r)^{-1}, r\right) .
\end{aligned}
$$

Thus $L\left(h_{2} \circ h_{1}\right)=L\left(h_{2}\right) \cdot L\left(h_{1}\right)$ on $(0,1)$. Since $L\left(h_{1}\right), L\left(h_{2}\right)$ and $L\left(h_{1} \circ h_{2}\right)$ are smooth maps on $[0,1], L\left(h_{2} \circ h_{1}\right)=L\left(h_{2}\right) \cdot L\left(h_{1}\right)$ on $[0,1]$. Thus $L$ is a group homomorphism. Suppose $L(h)=1$ for $h \in \operatorname{Ker} P$. Then $\left(h \circ \alpha^{-1}\right)(g H, r)$ $=\alpha^{-1}(g H, r)$ for $g \in G$ and $0<r<1$, and $h=1$ on $\pi^{-1}((0,1))$. Thus $h=1$ on $M$, and $L$ is a monomorphism.

Proposition 4.6. $L$ is a continuous map.

Proof. We shall use the notations in the proof of Proposition 4.1. Since $L$ is a group homomorphism, it is sufficient to show that $L: O \rightarrow Q$ is continuous. Let $I$ denote a closed interval $\left[0, \varepsilon^{2}\right],\left[\varepsilon^{2} / 2,1-\varepsilon^{2} / 2\right]$ or $\left[1-\varepsilon^{2}, 1\right]$. By Lemma 2.3 , it is sufficient to prove that $L_{I}: \mathrm{O} \stackrel{L}{\longrightarrow} Q \stackrel{j^{*}}{\longrightarrow} C^{\infty}(I, N(H) / H)$ is continuous, where $j: I \hookrightarrow[0,1]$ is an inclusion map.

First we shall consider the case $I=\left[0, \varepsilon^{2}\right]$. Let $L_{1}$ be the composition

$$
\begin{gathered}
O \stackrel{\left(k^{\circ} \phi_{0}^{-1} 1^{\circ} i\right)^{*}}{\longrightarrow} C^{\infty}\left([-\varepsilon, \varepsilon], p_{0, \varepsilon}^{-1}\left(U_{0}\right)\right) \\
\stackrel{\left(\sigma_{0}^{\circ} \rho_{1} \circ \phi_{0}, 1\right)_{*}}{\longrightarrow} C^{\infty}([-\varepsilon, \varepsilon], G),
\end{gathered}
$$

where $k: p_{0, \varepsilon}^{-1}\left(\bar{W}_{0}\right) \hookrightarrow M$ is an inclusion map. Then $L_{1}$ is continuous by Lemma 2.2. Note that $L_{1}(h)=b$.

Let $L_{2}: O \rightarrow C^{\infty}\left([-\varepsilon, \varepsilon],\left(N\left(H, K_{0}\right) / H\right)^{0}\right)$ be a map given by $L_{2}(h)=c$. We shall prove that $L_{2}$ is continuous. This is trivial in the case $N\left(H, K_{0}\right) / H$ $=Z_{2}$. Consider the case $\bar{K}_{0}=S U(n)(n \geq 2)$. In this case $V_{0}=C^{n}=R^{2 n}$ and $\bar{N}_{0}=U(1)=S O(2)$. Put $C_{0}^{\infty}\left([-\varepsilon, \varepsilon], V_{0}\right)=\left\{F \in C^{\infty}\left([-\varepsilon, \varepsilon], V_{0}\right) ; F(0)=0\right\}$, endowed with $C^{\infty}$ topology. Let $\Phi: C_{0}^{\infty}\left([-\varepsilon, \varepsilon], V_{0}\right) \rightarrow C^{\infty}\left([-\varepsilon, \varepsilon], R^{2}\right)$ be a map defined by $\Phi(F)=\left(\Phi\left(F^{1}\right), \Phi\left(F^{2}\right)\right)$, where $F=\left(F^{1}, \ldots, F^{2 n}\right)$ and $\Phi\left(F^{i}\right)$ is a map defined in Lemma 4.4. Then $\Phi$ is continuous by Lemma 4.4. Let $m: R^{2} \rightarrow M_{2}(R)$ denote a smooth map defined by

$$
m(x, y)=\left[\begin{array}{cc}
x & -y \\
y & x
\end{array}\right],
$$


where $M_{2}(R)$ denote the set of all $2 \times 2$ matrices over $R$. Let $L_{2}^{\prime}$ denote the composition

$$
\begin{gathered}
O \stackrel{\left(k^{\circ} \phi_{0,1}^{-1} \circ i\right)^{*}}{\longrightarrow} C^{\infty}\left([-\varepsilon, \varepsilon], p_{0,1}^{-1}\left(U_{0}\right)\right) \\
\stackrel{\left(\rho_{2} \circ \phi_{0}, 1\right)_{*}}{\longrightarrow} C^{\infty}\left([-\varepsilon, \varepsilon], D_{\varepsilon}\left(V_{0}\right)\right) .
\end{gathered}
$$

From Lemma 2.2, $L_{2}^{\prime}$ is continuous. Note that $L_{2}^{\prime}(h)=\tilde{h}_{2}$ and $L_{2}^{\prime}(O)$ is contained in $C_{0}^{\infty}\left([-\varepsilon, \varepsilon], V_{0}\right)$. Let $\hat{L}_{2}$ denote the composition

$$
\begin{aligned}
O \stackrel{L_{2}^{\prime}}{\longrightarrow} C_{0}^{\infty}\left([-\varepsilon, \varepsilon], V_{0}\right) \\
\stackrel{\Phi}{\longrightarrow} C^{\infty}\left([-\varepsilon, \varepsilon], R^{2}\right) \\
\stackrel{m_{*}}{\longrightarrow} C^{\infty}\left([-\varepsilon, \varepsilon], M_{2}(R)\right) .
\end{aligned}
$$

Then $\hat{L}_{2}(h)=\hat{c}$ and $\hat{L}_{2}$ is continuous. This implies that $L_{2}$ is continuous by using Lemma 2.2. Similarly we can see that $L_{2}$ is continuous in the other cases.

Let $\mu: G \times G / H \rightarrow G / H$ be a map defined by the left translation and let ८: $\left(N\left(H, K_{0}\right) / H\right)^{\circ} \hookrightarrow G / H$ be an inclusion map. Then the composition

$$
\begin{aligned}
\hat{L}: O & \stackrel{\left(L_{1}, \iota_{*}{ }^{\circ} L_{2}\right)}{\longrightarrow} C^{\infty}([-\varepsilon, \varepsilon], G) \times C^{\infty}([-\varepsilon, \varepsilon], G / H) \\
& \stackrel{\kappa}{\longrightarrow} C^{\infty}([-\varepsilon, \varepsilon], G \times G / H) \\
& \stackrel{\mu_{*}}{\longrightarrow} C^{\infty}([-\varepsilon, \varepsilon], G / H)
\end{aligned}
$$

is continuous by Lemma 2.2, where $\kappa$ is defined by $\kappa\left(f_{1}, f_{2}\right)(r)=\left(f_{1}(r), f_{2}(r)\right)$. Note that $\hat{L}(h)=b \cdot c=\hat{a}$ and $\hat{L}(O)$ is contained in $C_{e}^{\infty}([-\varepsilon, \varepsilon], N(H) / H)$. Here $C_{e}^{\infty}([-\varepsilon, \varepsilon], N(H) / H)$ denotes the set of all smooth even maps $f:[-\varepsilon, \varepsilon]$ $\rightarrow N(H) / H$, endowed with $C^{\infty}$ topology. Let $T: C_{e}^{\infty}([-\varepsilon, \varepsilon], N(H) / H) \rightarrow$ $C^{\infty}\left(\left[0, \varepsilon^{2}\right], N(H) / H\right)$ be a map defined by $T(f)(r)=f(\sqrt{r})$. By the same argument as in the proof in Lemma 2.4, we can prove that $T$ is continuous. Thus $L_{I}=T \circ L$ is continuous.

Now consider the case $I=\left[\varepsilon^{2} / 2,1-\varepsilon^{2} / 2\right] . \quad L_{I}$ is given by the composition

$$
\begin{aligned}
O \stackrel{k^{*}}{\longrightarrow} & C^{\infty}\left(\pi^{-1}(I), \pi^{-1}(I)\right) \\
\stackrel{\left(\alpha^{-1} \iota_{\iota}\right)^{*}}{\longrightarrow} & C^{\infty}\left(I, \pi^{-1}(I)\right) \\
\stackrel{\left(q_{2} \circ\right)_{*}}{\longrightarrow} & C^{\infty}(I, G / H),
\end{aligned}
$$

where $k: \pi^{-1}(I) \hookrightarrow M$ is an inclusion, $\iota: I \rightarrow G / H \times I$ is a map given by $\iota(r)$ $=(1 H, r)$ and $q_{2}: G / H \times I \rightarrow G / H$ is the projection on the first factor. Thus $L_{I}$ is continuous. We can see that $L_{I}$ is continuous in the case $I=\left[1-\varepsilon^{2}, 1\right]$ similarly as in the case $I=\left[0, \varepsilon^{2}\right]$, and this completes the proof of Proposition 4.6. 


\section{§5. Subgroups of the Topological Groups $Q$ and Ker $\mathbb{P}$}

In this section we shall consider subgroups $Q_{1}$ and $S$ of the topological groups $Q$ and $\operatorname{Ker} P$, respectively, such that $L(S)=Q_{1}$, and we shall prove that the inclusions $Q_{1} \hookrightarrow Q_{0}$ and $S \hookrightarrow \operatorname{Ker} P$ are homotopy equivalences, where $Q_{0}$ is the identity component of $Q$.

Put $Q_{1}=\left\{a \in Q_{0} ; a(r)=a(0)\right.$ for $0 \leq r \leq 1 / 4$ and $a(r)=a(1)$ for $\left.3 / 4 \leq r \leq 1\right\}$. Then $Q_{1}$ is a topological subgroup of $Q_{0}$. Let $i: Q_{1} \hookrightarrow Q_{0}$ be an inclusion.

Lemma 5.1. $i: Q_{1} \hookrightarrow Q_{0}$ is a homotopy equivalence.

Proof. Let $\sigma:[0,1] \rightarrow[0,1]$ be a smooth map such that

$$
\begin{array}{lll}
\sigma(r)=0 & \text { for } & 0 \leq r \leq 1 / 4 \\
\sigma(r)=1 & \text { for } & 3 / 4 \leq r \leq 1 .
\end{array}
$$

Let $\mu_{t}:[0,1] \rightarrow[0,1](0 \leq t \leq 1)$ be a smooth homotopy given by $\mu_{t}(r)=t \sigma(r)$ $+(1-t) r$. Since $\left(a \circ \mu_{t}\right)(i) \in\left(N(H) \cap N\left(K_{i}\right)\right) / H$ for $i=0,1, a \circ \mu_{t}$ is an element of $Q$. Define $q: Q_{0} \times[0,1] \rightarrow Q$ by $q(a, t)=a \circ \mu_{t}$. Let $\mu:[0,1] \rightarrow C^{\infty}([0,1],[0,1])$ be a map given by $\mu(t)=\mu_{t}$. Then it is easy to see that $\mu$ is continuous. Note that $q$ is given by the composition

$$
\begin{aligned}
Q_{0} \times[0,1] \stackrel{(1, \mu)}{\longrightarrow} Q_{0} \times C^{\infty}([0,1],[0,1]) \\
\stackrel{\text { comp }}{\longrightarrow} C^{\infty}([0,1], N(H) / H),
\end{aligned}
$$

where comp is given by comp $(a, f)=a \circ f$. By Lemma $2.2(6), q$ is continuous. Then $q\left(Q_{0} \times[0,1]\right)$ is contained in $Q_{0}$. Let $q_{t}: Q_{0} \rightarrow Q_{0}$ be a map given by $q_{t}(a)=q(a, t)$. Since $\mu_{1}=\sigma, q_{1}\left(Q_{0}\right)$ is contained in $Q_{1}$. Thus $q$ is a homotopy between $q_{0}=1_{Q_{0}}$ and $q_{1}=i \circ q_{1}$. Note that $q_{t}\left(Q_{1}\right)$ is contained in $Q_{1}$ for any $t$. Then $q: Q_{1} \times[0,1] \rightarrow Q_{1}$ is a homotopy between $1_{Q_{1}}$ and $q_{1} \circ i$. Therefore Lemma 5.1 follows.

Put $S=L^{-1}\left(Q_{1}\right) \subset \operatorname{Ker} P$. Let $\iota: S \hookrightarrow \operatorname{Ker} P$ be an inclusion.

Lemma 5.2. $\quad$ : $S \hookrightarrow \operatorname{Ker} P$ is a homotopy equivalence.

Proof. Put $a=L\left(h^{-1}\right)$ for $h \in \operatorname{Ker} P$. Let $h_{t}: M \rightarrow M(0 \leq t \leq 1)$ be a map as follows: $h_{t}$ is given on $\pi^{-1}((0,1))$ by the composition $\pi^{-1}((0,1)) \stackrel{\alpha}{\longrightarrow} G / H$ $\times(0,1) \stackrel{\hat{h}_{t}}{\longrightarrow} G / H \times(0,1) \stackrel{\alpha^{-1}}{\longrightarrow} \pi^{-1}((0,1))$, where $\hat{h}_{t}$ is defined by $\hat{h}_{t}(g H, r)$ $=\left(g \cdot q_{t}(a)(r), r\right) . \quad h_{t}\left(g K_{i}\right)=g a(i) \cdot K_{i}(i=0,1)$ for $g \in G$. Here we need the following 
Assertion 5.3. $\quad h_{t}$ is a smooth map for any $t$.

Proof. By the definition, $h_{t}$ is smooth on $\pi^{-1}((0,1))$. We shall prove that $h_{t}$ is smooth on $\pi^{-1}(0)$. Let $a_{0}$ be an element of $G$ such that $a_{0} H=a(0)$ and $a_{0} \in N(H) \cap N\left(K_{0}\right)$. For $[g, 0] \in p_{0,1 / 2}^{-1}\left(1 K_{0}\right),\left(p_{0,1 / 2}^{\circ} h\right)([g, 0])=\pi_{0}\left(g a_{0}\right)$ $=\pi_{0}\left(a_{0}\right) \in a_{0} U_{0}$. Then there exists a neighborhood $W_{0}$ of $1 K_{0}$ in $G / K_{0}$ such that $\left(p_{0,1 / 2} \circ\right)\left(p_{0,1 / 2}^{-1}\left(\bar{W}_{0}\right)\right)$ is contained in $a_{0} U_{0}$. For $\left[g, r e_{0}\right] \in p_{0,1 / 2}^{-1}\left(\bar{W}_{0}\right)$ and $0 \leq t \leq 1$,

$$
\begin{aligned}
\left(p_{0,1 / 2} \circ h_{t}\right)\left(\left[g, r e_{0}\right]\right) & =\bar{\pi}_{0}\left(g q_{t}(a)\left(r^{2}\right)\right) \\
& =\bar{\pi}_{0}\left(g a\left((1-t) r^{2}\right)\right) \\
& =\left(p_{0,1 / 2} \circ h\right)\left(\left[g, \sqrt{1-\imath} r e_{0}\right]\right)
\end{aligned}
$$

which is contained in $\left(p_{0,1 / 2} \circ\right)\left(p_{0,1 / 2}^{-1}\left(\bar{W}_{0}\right)\right) \subset a_{0} U_{0}$. Then $h_{t}\left(p_{0,1 / 2}^{-1}\left(g \bar{W}_{0}\right)\right)$ is contained in $p_{0,1 / 2}^{-1}\left(g a_{0} U_{0}\right)$ for $g \in G$ and $0 \leq t \leq 1$.

Let $\tilde{h}: W_{0} \times D_{1 / 2}\left(V_{0}\right) \rightarrow U_{0} \times D_{1 / 2}\left(V_{0}\right)$ be a map given by $\tilde{h}=\phi_{0, g a_{0}} \circ h^{\circ} \phi_{0, g}^{-1}$ for $g \in G$. Let $\rho_{1}: U_{0} \times D_{1 / 2}\left(V_{0}\right) \rightarrow U_{0}$ and $\rho_{2}: U_{0} \times D_{1 / 2}\left(V_{0}\right) \rightarrow D_{1 / 2}\left(V_{0}\right)$ be the projections on the first factor and the second factor respectively. Put $g^{\prime}=g a_{0}$ and put $\tilde{h}^{i}=\rho_{i} \circ \tilde{h}$ for $i=0,1$. Then $\tilde{h}^{i}$ is a smooth map and

$$
\begin{aligned}
& \tilde{h}^{1}\left(x, r k e_{0}\right)=g^{\prime-1} g \sigma_{0}(x) k \cdot \bar{\pi}_{0}\left(a\left(r^{2}\right)\right), \\
& \tilde{h}^{2}\left(x, r k e_{0}\right)=\sigma_{0, g^{\prime}}\left(g \sigma_{0}(x) k \cdot \bar{\pi}_{0}\left(a\left(r^{2}\right)\right)^{-1} g \sigma_{0}(x) k a\left(r^{2}\right) \cdot r e_{0}\right.
\end{aligned}
$$

for $x \in W_{0}$ and $k \in K_{0}$, where $\bar{\pi}_{0}: G / H \rightarrow G / K_{0}$ is the natural projection. Put $\tilde{h}_{t}^{i}=\rho_{i} \circ \phi_{0, g^{\circ}} \circ h_{t}^{\circ} \phi_{0, g}^{-1}$ for $i=0,1$. Then

$$
\begin{aligned}
& \tilde{h}_{t}^{1}\left(x, r k e_{0}\right)=g^{\prime-1} g \sigma_{0}(x) k \cdot \bar{\pi}_{0}\left(a\left(\mu_{t}\left(r^{2}\right)\right)\right), \\
& \tilde{h}_{t}^{2}\left(x, r k e_{0}\right)=\sigma_{0, g^{\prime}}\left(g \sigma_{0}(x) k \cdot \bar{\pi}_{0}\left(a\left(\mu_{t}\left(r^{2}\right)\right)\right)^{-1} g \sigma_{0}(x) k a\left(\mu_{t}\left(r^{2}\right)\right) \cdot r e_{0}\right.
\end{aligned}
$$

for $x \in W_{0}$ and $k \in K_{0}$.

Since $\sigma\left(r^{2}\right)=0$ for $r \leq 1 / 2, \mu\left(r^{2}, t\right)=(1-t) r^{2}$ for $0 \leq r \leq 1 / 2$. Then $\tilde{h}_{t}^{1}(x, v)$ $=\tilde{h}^{1}(x, \sqrt{1-t} v)$ for $0 \leq t \leq 1$ and $\tilde{h}_{t}^{2}(x, v)=1 / \sqrt{1-t} \tilde{h}^{2}(x, \sqrt{1-t} v)$ for $0 \leq t<1$. Thus $\tilde{h}_{t}^{1}(0 \leq t \leq 1)$ and $\tilde{h}_{t}^{2}(0 \leq t<1)$ are smooth maps.

By the Taylor formula (c.f. J. Dieudonné $[5$, Chapter VIII, $(8,14,3)]$ ), we have

$$
\tilde{h}^{2}(x, v)=\tilde{h}^{2}(x, 0)+\left(\int_{0}^{1}\left(D \tilde{h}^{2}\right)(x, \zeta v) d \zeta\right) v,
$$

where $D \tilde{h}^{2}$ is the derivative of $\tilde{h}^{2}$. Since $\tilde{h}^{2}(x, 0)=0$,

$$
\tilde{h}_{t}^{2}(x, v)=\left(\int_{0}^{1}\left(D \tilde{h}^{2}\right)(x, \sqrt{1-t} \zeta v) d \zeta\right) v \quad \text { for } \quad 0 \leq t<1
$$


Then $\tilde{h}_{1}^{2}(x, v)=\lim _{t \rightarrow 1} \tilde{h}_{t}^{2}(x, v)=\left(D \tilde{h}^{2}\right)(x, 0) v$, and $\tilde{h}_{1}^{2}$ is a smooth map. Therefore $h_{t}$ is smooth on $\pi^{-1}(0)$ for any $0 \leq t \leq 1$. Similarly we can prove that $h_{t}$ is smooth on $\pi^{-1}(1)$, and Assertion 5.3 follows.

Proof of Lemma 5.2 continued. Let $\bar{q}: \operatorname{Ker} P \times[0,1] \rightarrow \operatorname{Ker} P$ be a map defined by $\bar{q}(h, t)=h_{t}$. By Assertion 5.3, $h_{t}$ and $h_{t}^{-1}$ are smooth maps, and $\bar{q}$ is a well defined map. Next we shall prove that $\bar{q}$ is continuous. Let $W_{i}$ be a neighborhood of $1 K_{i}$ in $G / K_{i}$ satisfying $\bar{W}_{i} \subset U_{i}$ for $i=0,1$. Put $O=\{h \in \operatorname{Ker} P$; $h\left(p_{i, 1 / 2}^{-1}\left(\bar{W}_{i}\right)\right) \subset p_{i, 1 / 2}^{-1}\left(U_{i}\right)$ for $\left.i=0,1\right\}$. Then $O$ is an open neighborhood of $1_{M}$ in $\operatorname{Ker} P$. For $h \in O, g \in G$ and $0 \leq t \leq 1, h_{t}\left(p_{i, 1 / 2}^{-1}\left(g \bar{W}_{i}\right)\right)$ is contained in $p_{i, 1 / 2}^{-1}\left(g U_{i}\right)(i=0,1)$. Let $W_{2}$ be an open neighborhood of $1 H$ in $G / H$ satisfying $\bar{W}_{2} \subset U_{2}$. Let $C$ be one of the sets $\left\{p_{i, 1 / 2}^{-1}\left(g \bar{W}_{i}\right)(i=0,1, g \in G), \alpha^{-1}\left(g \bar{W}_{2}\right.\right.$ $\times[1 / 5,4 / 5])(g \in G)\} . \quad$ By Lemma 2.3, it is sufficient to show that the composition $\bar{q}_{C}: O \times[0,1] \stackrel{\bar{q}}{\longrightarrow} \operatorname{Ker} P \stackrel{j_{C}^{*}}{\longrightarrow} C^{\infty}(C, M)$ is continuous for any $C$, where $j_{C}: C \hookrightarrow M$ is an inclusion map.

First consider the case $C=p_{0,1 / 2}^{-1}\left(g \bar{W}_{0}\right)$. Let $v_{1}: C^{\infty}\left(\bar{W}_{0} \times D_{1 / 2}\left(V_{0}\right), U_{0}\right) \times$ $[0,1] \rightarrow C^{\infty}\left(\bar{W}_{0} \times D_{1 / 2}\left(V_{0}\right), U_{0}\right)$ be a map given by $v_{1}(f, t)(x, v)=f(x, \sqrt{1-t} v)$. Let $v_{2}: C^{\infty}\left(\bar{W}_{0} \times D_{1 / 2}\left(V_{0}\right), D_{1 / 2}\left(V_{0}\right)\right) \times[0,1] \rightarrow C^{\infty}\left(\bar{W}_{0} \times D_{1 / 2}\left(V_{0}\right), D_{1 / 2}\left(V_{0}\right)\right)$ be a map given by $v_{2}(f, t)(x, v)=\left(\int_{0}^{1}(D f)(x, \sqrt{1-t} \zeta v) d \zeta\right)(v)$. It is easy to see that $v_{1}$ and $v_{2}$ are continuous. Note that $\bar{q}_{C}$ is the composition

$$
\begin{aligned}
O \times[0,1] \stackrel{\left(j_{C}^{*}, 1\right)}{\longrightarrow} C^{\infty}\left(C, p_{0,1 / 2}^{-1}\left(g U_{0}\right)\right) \times[0,1] \\
\stackrel{\left(\left(\phi_{0}, g\right)_{*}^{*}(\phi 0, g)^{*}, 1\right)}{\longrightarrow} C^{\infty}\left(\bar{W}_{0} \times D_{1 / 2}\left(V_{0}\right), U_{0} \times D_{1 / 2}\left(V_{0}\right)\right) \times[0,1] \\
\stackrel{\left(\left(\rho_{1}\right)_{*},\left(\rho_{2}\right)_{*}, 1\right)}{\longrightarrow} C^{\infty}\left(\bar{W}_{0} \times D_{1 / 2}\left(V_{0}\right), U_{0}\right) \\
\quad \times C^{\infty}\left(\bar{W}_{0} \times D_{1 / 2}\left(V_{0}\right), D_{1 / 2}\left(V_{0}\right)\right) \times[0,1] \\
\stackrel{v}{\longrightarrow} C^{\infty}\left(\bar{W}_{0} \times D_{1 / 2}\left(V_{0}\right), U_{0}\right) \times C^{\infty}\left(\bar{W}_{0} \times D_{1 / 2}\left(V_{0}\right), D_{1 / 2}\left(V_{0}\right)\right) \\
\stackrel{\kappa}{\longrightarrow} C^{\infty}\left(\bar{W}_{0} \times D_{1 / 2}\left(V_{0}\right), U_{0} \times D_{1 / 2}\left(V_{0}\right)\right) \\
\stackrel{\left(\phi_{0, g}^{-1}\right)_{*^{\circ}}\left(\phi_{0, g}^{-1}\right)^{*}}{\longrightarrow} C^{\infty}\left(C, p_{0,1 / 2}^{-1}\left(g U_{0}\right)\right) C C^{\infty}(C, M) .
\end{aligned}
$$

Here $v$ is given by $v\left(f_{1}, f_{2}, t\right)=\left(v_{1}\left(f_{1}, t\right), v_{2}\left(f_{2}, t\right)\right)$ and $\kappa$ is the map defined in Lemma 2.2 (5). Then $\bar{q}_{C}$ is continuous by Lemma 2.2 .

Next consider the case $C=\alpha^{-1}\left(g \bar{W}_{2} \times[1 / 5,4 / 5]\right)$. Let $m: N(H) / H \times G / H$ $\rightarrow G / H$ be a map defined by $m(n H, g H)=(g n) H$ and $p_{2}: G / H \times[1 / 5,4 / 5] \rightarrow[0,1]$ be a map given by $p_{2}(g H, r)=r$. Let $\delta: Q_{0} \rightarrow Q_{0}$ be a map given by $\delta(a)=a^{-1}$. Then the map $\bar{q}_{C}$ is the composition 


$$
\begin{aligned}
O \times[0,1] & \stackrel{(L, 1)}{\longrightarrow} Q_{0} \times[0,1] \stackrel{\delta \circ q}{\longrightarrow} Q_{0} \stackrel{p_{2}^{*}}{\longrightarrow} C^{\infty}(G / H \times[1 / 5,4 / 5], N(H) / H) \\
& \stackrel{\left(1_{G^{\prime} H \times[1 / 5,4 / 5}\right)_{1}}{\longrightarrow} C^{\infty}(G / H \times[1 / 5,4 / 5], \\
N(H) / H \times G / H \times[1 / 5,4 / 5]) & \\
& \stackrel{m_{*}}{\longrightarrow} C^{\infty}(G / H \times[1 / 5,4 / 5], G / H \times[1 / 5,4 / 5]) \\
& \stackrel{\left(\alpha \circ{ }^{*}\right)^{*}\left(\alpha^{-1}\right)_{*}}{\longrightarrow} C^{\infty}\left(C, \alpha^{-1}(G / H \times[1 / 5,4 / 5])\right) \hookrightarrow C^{\infty}(C, M),
\end{aligned}
$$

which is continuous because $L$ and $q$ are continuous.

Similarly as in the case $C=p_{0,1 / 2}^{-1}\left(g \bar{W}_{0}\right)$, we can see that $\bar{q}_{C}$ is continuous in the case $C=p_{1,1 / 2}^{-1}\left(g \bar{W}_{1}\right)$. Thus $\bar{q}$ is continuous. Since $q_{1}\left(Q_{0}\right) \subset Q_{1}, \bar{q}_{1}(\operatorname{Ker} P)$ $\subset S$. Therefore $\bar{q}$ is a homotopy between $\bar{q}_{0}=1_{\text {Ker } P}$ and $\bar{q}_{1}=\iota^{\circ} \bar{q}_{1}$. Since $q\left(Q_{1} \times[0,1]\right) \subset Q_{1}, \bar{q}(S \times[0,1]) \subset S$. Then $\bar{q}: S \times[0,1] \rightarrow S$ is a homotopy between $1_{S}$ and $\bar{q}_{1} \circ$. Thus $\iota$ is a homotopy equivalence, and this completes the proof of Lemma 5.2.

\section{§6. Proof of Theorem}

In this section, we shall see that $L: S \rightarrow Q_{1}$ is an isomorphism between topological groups, and we shall prove our Theorem.

Proposition 6.1. $L: S \rightarrow Q_{1}$ is an isomorphism between topological groups.

Before the proof of Proposition 6.1, we begin with some lemmas. For any topological subgroup $K$ of $G, K^{0}$ denotes the identity component of $K$.

Lemma 6.2. For any $a \in N\left(K_{0}\right)^{0} \cap N(H)$, there exist $a^{\prime} \in N\left(H^{0}\right) \cap K_{0}^{0}$ and $n \in \operatorname{Cent}\left(K_{0}^{0}\right)$ such that $a=n \cdot a^{\prime}$, where Cent $\left(K_{0}^{0}\right)$ is the centralizer of $K_{0}^{0}$ in $G$.

Proof. Since $N\left(K_{0}\right)^{0}$ is a compact connected Lie group, there exist a torus group $T$ and a simply connected semi-simple compact Lie group $G^{\prime}$ such that $\hat{N}_{0}=T \times G^{\prime}$ is a finite covering group of $N\left(K_{0}\right)^{0}$ (c.f. L. Pontrjagin [8, §64]). Let $q_{0}: \widehat{N}_{0} \rightarrow N\left(K_{0}\right)^{0}$ be the covering projection. Put $\hat{K}_{0}=q_{0}^{-1}\left(K_{0}^{0}\right)$. Since $K_{0}^{0}$ is a normal subgroup of $N\left(K_{0}\right)^{0}, \hat{K}_{0}$ is a normal subgroup of $\hat{N}_{0}$. Then $\hat{K}_{0}^{0}$ is also a normal subgroup of $\hat{N}_{0}$. Here we need the following

Assertion 6.3. There exists a closed normal subgroup $K_{0}^{\prime}$ of $\hat{N}_{0}$ such that $\hat{N}_{0}$ is isomorphic to the product group $\hat{K}_{0}^{0} \times K_{0}^{\prime}$ as a Lie group.

Proof. There exist simply connected simple Lie groups $G_{i}(1 \leq i \leq r)$ such that $G^{\prime}=G_{1} \times \cdots \times G_{r}$. Since $\hat{K}_{0}^{0}$ is a compact connected Lie group, there exist 
simply connected simple Lie groups $K_{j}(1 \leq j \leq s)$ and a torus group $T^{\prime}$ such that $\tilde{K}_{0}=T^{\prime} \times K_{1} \times \cdots \times K_{s}$ is a finite covering of $\hat{K}_{0}^{0}$. Let $p_{0}: \widetilde{K}_{0} \rightarrow \hat{K}_{0}^{0}$ be the covering projection. Let $\rho_{i}: \hat{N}_{0}=T \times G_{1} \times \cdots \times G_{r} \rightarrow G_{i}$ be a projection on the direct factor $G_{i}(1 \leq i \leq r)$. Since $\hat{K}_{0}^{0}$ is a normal subgroup of $\hat{N}_{0}, \rho_{i}\left(\hat{K}_{0}^{0}\right)$ is a normal subgroup of $G_{i}$. Since $G_{i}$ is a simple Lie group, $\rho_{\imath}\left(\widehat{K}_{0}^{0}\right)=G_{i}$ or $\{1\}$. If $\rho_{i}\left(\hat{K}_{0}^{0}\right)=G_{i}, \rho_{i}\left(p_{0}\left(K_{j}\right)\right)$ is a normal subgroup of $G_{i}$. Thus $\rho_{i}\left(p_{0}\left(K_{j}\right)\right)=G_{i}$ or $\{1\}$, for $1 \leq i \leq r, 1 \leq j \leq s$.

Put $\rho_{i}^{\prime}=\rho_{i}^{\circ} p_{0}$. If $\rho_{i}^{\prime}\left(K_{j_{1}}\right)=\rho_{i}^{\prime}\left(K_{j_{2}}\right)\left(j_{1} \neq j_{2}\right)$, then $\rho_{i}^{\prime}\left(g_{1}\right) \cdot \rho_{i}^{\prime}\left(g_{2}\right)=\rho_{i}^{\prime}\left(g_{1}\right.$. $\left.g_{2}\right)=\rho_{i}^{\prime}\left(g_{2} \cdot g_{1}\right)=\rho_{i}^{\prime}\left(g_{2}\right) \cdot \rho_{i}^{\prime}\left(g_{1}\right)$ for $g_{1} \in K_{j_{1}}, g_{2} \in K_{j_{2}}$. Then $\rho_{i}^{\prime}\left(K_{j_{1}}\right)$ is a commutative normal subgroup of $G_{i}$, and $\rho_{i}^{\prime}\left(K_{j_{1}}\right)=\{1\}$. If $\rho_{i}^{\prime}\left(K_{j}\right)=G_{i}$, then $\rho_{i}^{\prime}\left(T^{\prime}\right)$ is a normal subgroup of $G_{i}$, hence $\rho_{i}^{\prime}\left(T^{\prime}\right)=\{1\}$. Therefore, if $\rho_{i}^{\prime}\left(K_{j}\right)$ $=G_{i}$, then $\rho_{i}^{\prime}\left(T^{\prime}\right)=\{1\}$ and $\rho_{i}^{\prime}\left(K_{n}\right)=\{1\}$ for $n \neq j$.

Assume $\rho_{i_{1}}^{\prime}\left(K_{j}\right)=G_{i_{1}}$ and $\rho_{i_{2}}^{\prime}\left(K_{j}\right)=G_{i_{2}}$ for $i_{1} \neq i_{2}$. Let $\rho^{\prime}: \widetilde{K}_{0} \rightarrow G_{i_{1}} \times G_{i_{2}}$ be a map defined by $\rho^{\prime}(k)=\left(\rho_{i_{1}}^{\prime}(k), \rho_{i_{2}}^{\prime}(k)\right)$. Since $\hat{K}_{0}^{0}$ is a normal subgroup of $\hat{N}_{0}$ and $\rho^{\prime}\left(\tilde{K}_{0}\right)=\rho^{\prime}\left(K_{j}\right), \rho^{\prime}\left(K_{j}\right)$ is a normal subgroup of $G_{i_{1}} \times G_{i_{2}}$. Then, for $x, y \in K_{j}$, there exists $k \in K_{j}$ such that $\left(\rho_{i_{1}}^{\prime}(x), 1\right) \rho^{\prime}(y)\left(\rho_{i_{1}}^{\prime}(x)^{-1}, 1\right)=\rho^{\prime}(k)$. Then $\rho_{i_{1}}^{\prime}\left(x y x^{-1}\right)=\rho_{i_{1}}^{\prime}(x) \rho_{i_{1}}^{\prime}(y) \rho_{i_{1}}^{\prime}(x)^{-1}=\rho_{i_{1}}^{\prime}(k)$ and $\rho_{i_{2}}^{\prime}(y)=\rho_{i_{2}}^{\prime}(k)$. Since $K_{j}$, $G_{i_{n}}(n=1,2)$ are simply connected simple Lie groups, $\rho_{i_{n}}^{\prime}: K_{j} \rightarrow G_{i_{n}}$ is an isomorphism between the Lie groups. Thus $x y x^{-1}=k=y$ for any $x, y \in K_{j}$, and $K_{j}$ must be a commutative Lie group, which is a contradiction since $K_{j}$ is a simple Lie group.

Thus we may assume that $\rho_{j}^{\prime}\left(K_{j}\right)=G_{j}$ and $\rho_{i}^{\prime}\left(K_{j}\right)=\{1\}(i \neq j)$ for $1 \leq j \leq s$, $1 \leq i \leq r$. For $i>s, \rho_{i}\left(\hat{K}_{0}^{0}\right)=\rho_{i}^{\prime}\left(\widetilde{K}_{0}\right)=\rho_{i}^{\prime}\left(T^{\prime}\right)$ which is a commutative normal subgroup of $G_{i}$, hence $\rho_{i}^{\prime}\left(T^{\prime}\right)=\{1\}$. Then $p_{0}\left(T^{\prime}\right)$ is a subgroup of $T$, and there exists a torus subgroup $S$ of $T$ such that $T=p_{0}\left(T^{\prime}\right) \times S$. Put $K^{\prime}=S \times G_{s+1} \times \cdots \times$ $G_{r}$. Then $\hat{N}_{0}=\hat{K}_{0}^{0} \times K_{0}^{\prime}$, and Assertion 6.3 follows.

Proof of Lemma 6.2 continued. By Assertion 6.3, there exists a closed normal subgroup $K_{0}^{\prime}$ of $\hat{N}_{0}$ such that $\widehat{N}_{0}=\widehat{K}_{0}^{0} \times K_{0}^{\prime}$. Since $K_{0}^{0}$ is a connected group, $q_{0}\left(\hat{K}_{0}^{0}\right)=K_{0}^{0}$. Then $N\left(K_{0}\right)^{0}=q_{0}\left(\hat{N}_{0}\right)=q_{0}\left(\hat{K}_{0}^{0}\right) \cdot q_{0}\left(K_{0}^{\prime}\right)=K_{0}^{0} \cdot q_{0}\left(K_{0}^{\prime}\right)$. Note that $q_{0}\left(K_{0}^{\prime}\right)$ is contained in $\operatorname{Cent}\left(K_{0}^{0}\right)$. Thus, for $a \in N\left(K_{0}\right)^{0} \cap N(H)$, there exists $a^{\prime} \in K_{0}^{0}$ and $n \in \operatorname{Cent}\left(K_{0}^{0}\right)$ such that $a=a^{\prime} \cdot n$. Since $N(H) \subset N\left(H^{0}\right)$ and $H^{0} \subset K_{0}^{0}, H^{0}=a H^{0} a^{-1}=a^{\prime} n H^{0} n^{-1} a^{\prime-1}=a^{\prime} H^{0} a^{\prime-1}$. Thus $a^{\prime} \in N\left(H^{0}\right)$ and Lemma 6.2 follows.

For $a \in Q_{1}$, we define a map $h: M \rightarrow M$ as follows:

$$
h\left(\alpha^{-1}(g H, r)\right)=\alpha^{-1}\left(\left(g a(r)^{-1}, r\right)\right) \quad \text { for }(g H, r) \in G / H \times(0,1),
$$




$$
h([g, 0])=\left[g a(i)^{-1}, 0\right] \quad \text { for }[g, 0] \in \pi^{-1}(i) \quad(i=0,1) .
$$

Lemma 6.4. $h$ is a smooth map.

Proof. Choose $a_{0} \in\left(N(H) \cap N\left(K_{0}\right)\right)^{0} \subset N(H)^{0} \cap N\left(K_{0}\right)^{0}$ such that $a(0)^{-1}$ $=a_{0} H$. There exists a neighborhood $W_{0}$ of $1 K_{0}$ in $G / K_{0}$ such that $\pi_{0}^{-1}\left(W_{0}\right) \cdot a_{0}$ is contained in $a_{0} \cdot \pi_{0}^{-1}\left(U_{0}\right)$. Since $a(r)=a(0)$ for $0 \leq r \leq 1 / 4, h\left(p_{0,1 / 2}^{-1}\left(g W_{0}\right)\right)$ is contained in $p_{0,1 / 2}^{-1}\left(g a_{0} U_{0}\right)$. Let $\tilde{h}_{1}: W_{0} \times D_{1 / 2}\left(V_{0}\right) \rightarrow U_{0}$ be a map given by the composition $\rho_{1} \circ \phi_{0, g a_{0}} \circ h^{\circ} \phi_{0, g}^{-1}$, and let $\tilde{h}_{2}: W_{0} \times D_{1 / 2}\left(V_{0}\right) \rightarrow D_{1 / 2}\left(V_{0}\right)$ be a map given by the composition $\rho_{2} \circ \phi_{0, g a_{0}} \circ h^{\circ} \phi_{0, g}^{-1}$. Note that

$$
\begin{aligned}
\left(h \circ \phi_{0, g}^{-1}\right)\left(\left(x, r k e_{0}\right)\right) & =h\left(\left[g \sigma_{0}(x) k, r e_{0}\right]\right) \\
& =h\left(\alpha^{-1}\left(\left(g \sigma_{0}(x) k H, r^{2}\right)\right)\right) \\
& =\alpha^{-1}\left(\left(g \sigma_{0}(x) k a_{0} H, r^{2}\right)\right) \\
& =\left[g \sigma_{0}(x) k a_{0}, r e_{0}\right]
\end{aligned}
$$

for $x \in W_{0}, k \in K_{0}, 0<r \leq 1 / 2$. Since $a_{0} \in N\left(K_{0}\right), k a_{0} K_{0}=a_{0} K_{0}$. Then

$$
\begin{aligned}
& \tilde{h}_{1}(x, v)=a_{0}^{-1} \sigma_{0}(x) a_{0} K_{0} \text { for }(x, v) \in W_{0} \times D_{1 / 2}\left(V_{0}\right), \text { and } \\
& \tilde{h}_{2}\left(x, r k e_{0}\right)=\sigma_{0, g a_{0}}\left(g \sigma_{0}(x) a_{0} K_{0}\right)^{-1} g \sigma_{0}(x) k a_{0} \cdot r e_{0}
\end{aligned}
$$

for $x \in W_{0}, k \in K_{0}, 0 \leq r \leq 1 / 2$. Thus $\tilde{h}_{1}$ is a smooth map and $\tilde{h}_{2}$ is smooth on $W_{0} \times\left(D_{1 / 2}\left(V_{0}\right)-0\right)$. We shall prove that $\tilde{h}_{2}$ is smooth on $W_{0} \times 0$, hence $h$ is smooth on $\pi^{-1}(0)$. This is trivial in the case $\operatorname{dim} S\left(V_{0}\right)=0$.

Let $\xi_{a_{0}, g}: W_{0} \rightarrow G$ be a map given by $\xi_{a_{0}, g}(x)=\sigma_{0, g a_{0}}\left(g \sigma_{0}(x) a_{0} K_{0}\right)^{-1} g \sigma_{0}(x)$. Then $\xi_{a_{0}, g}$ is a smooth map. By Lemma 6.2, there exist $a_{0}^{\prime} \in N\left(H^{0}\right) \cap K_{0}^{0}$ and $n \in \operatorname{Cent}\left(K_{0}^{0}\right) \quad$ such that $a_{0}=n a_{0}^{\prime}$. Then $\tilde{h}_{2}\left(x, r k e_{0}\right)=\xi_{a_{0}, g}(x) k n a_{0}^{\prime} \cdot r k e_{0}$ $=\xi_{a_{0}, g}(x) n k a_{0}^{\prime} \cdot r e_{0}$ for $x \in W_{0}, k \in K_{0}^{0}$ and $0 \leq r \leq 1 / 2$. Note that $N\left(H^{0}\right) \cap K_{0}^{0}$ $=N\left(H^{0}, K_{0}^{0}\right)$.

Assertion 6.5. For $a \in N\left(H^{0}, K_{0}^{0}\right)$, let $\psi_{a}: D\left(V_{0}\right) \rightarrow D\left(V_{0}\right)$ be a map defined by $\psi_{a}\left(r k e_{0}\right)=r k a e_{0}$ for $0 \leq r \leq 1, k \in K$. Then $\psi_{a}$ is a diffeomorphism. Moreover, let $\psi: N\left(H^{0}, K_{0}^{0}\right) \rightarrow \operatorname{Diff}^{\infty}\left(D\left(V_{0}\right)\right)$ be a map given by $\psi(a)=\psi_{a}$, then $\psi$ is continuous.

Proof. If $\operatorname{dim} S\left(V_{0}\right)=0$, then $K_{0}^{0} \subset H$ and $\psi_{a}=1_{D\left(V_{0}\right)}$. In this case, the proof is trivial. We assume $\operatorname{dim} S\left(V_{0}\right)>0$. Since $S\left(V_{0}\right)=K_{0} / H$ is connected, $K_{0}^{0}$ acts transitively on $S\left(V_{0}\right)$. Let $L$ be the ineffective kernel of the action $K_{0}^{0} \times S\left(V_{0}\right) \rightarrow S\left(V_{0}\right)$. Put $\bar{K}=K_{0}^{0} / L$ and $\bar{H}=\left(H \cap K_{0}^{0}\right) / L$. Then $\bar{K}$ acts transitively and effectively on $S\left(V_{0}\right)$ and $\bar{H}$ is an isotropy subgroup of this action. By Lemma $4.3, \bar{K}, \bar{H}$ and $N(\bar{H}, \bar{K}) / \bar{H}$ are $G, H$ and $N(H) / H$ in Lemma 4.3, respec- 
tively. Hence $\bar{H}$ is connected. Since the identity component of $H \cap K_{0}^{0}$ is $H^{0}$, $\bar{H}=H^{0} \cdot L / L$. For $a \in N\left(H^{0}, K_{0}^{0}\right)$, the left coset $a L$ is an element of $N(\bar{H}, \bar{K})$. Then $a$ defines an element $\tilde{a} \in N(\bar{H}, \bar{K}) / \bar{H}$. Note that $\psi_{a}\left(r k e_{0}\right)=r k a e_{0}=r k \tilde{a} e_{0}$ for $0 \leq r \leq 1, k \in K_{0}^{0}$.

Consider the case $\bar{K}=S U(n)(n \geq 2), \bar{H}=S U(n-1)$ and $N(\bar{H}, \bar{K}) / \bar{H}=U(1)$. In this case, $V_{0}=C^{n}$ and $U(1)$ acts on $V_{0}$ as a scalar multiplication. Thus $\psi_{a}\left(r k e_{0}\right)=\tilde{a} \cdot r k e_{0}$ for $r k e_{0} \in D\left(V_{0}\right)$. Hence $\psi_{a}$ is a diffeomorphism. It is easy to see that $\psi$ is continuous.

Next consider the case $\bar{K}=S p(n)(n \geq 1), \bar{H}=S p(n-1)$ and $N(\bar{H}, \bar{K}) / \bar{H}$ $=S p(1)$. In this case, $V_{0}=H^{n}$ and $S p(1)$ acts on $V_{0}$ as a scalar multiplication on the right. Then $\psi_{a}(v)=v \cdot \tilde{a}$ for $v \in D\left(V_{0}\right)$, hence $\psi_{a}$ is a diffeomorphism and $\psi$ is continuous. Similarly we can see that $\psi_{a}$ is a diffeomorphism and $\psi$ is continuous in the other cases, and Assertion 6.5 follows.

Proof of Lemma 6.4 continued. Since $\tilde{h}_{2}(x, v)=\xi_{a_{0}, g}(x) n \cdot \psi_{a_{0}^{\prime}}(v)$, by Assertion 6.5, $\tilde{h}_{2}$ is a smooth map. Thus $\tilde{h}_{1}$ and $\tilde{h}_{2}$ are smooth maps, hence $h$ is smooth on $\pi^{-1}(0)$. Similarly we can see that $h$ is smooth on $\pi^{-1}(1)$. By the definition, $h$ is smooth on $\pi^{-1}((0,1))$, and this completes the proof of Lemma 6.4 .

Let $\hat{L}(a)$ be a smooth map $h: M \rightarrow M$ in Lemma 6.4, for $a \in Q_{1}$. Since $\hat{L}\left(a^{-1}\right)=\hat{L}(a)^{-1}, h$ is a diffeomorphism of $M$. By the definition, $h$ is an equivariant map. Thus we have a map $\hat{L}: Q_{1} \rightarrow \operatorname{Diff}_{G}^{\infty}(M)$. Note that $\hat{L}$ is an abstract group homomorphism.

Lemma 6.6. $\hat{L}: Q_{1} \rightarrow \operatorname{Diff}_{G}^{\infty}(M)$ is continuous.

Proof. Let $W_{i}$ be a neighborhood of $1 K_{i}$ in $G / K_{i}$ such that $\bar{W}_{i} \subset U_{i}$ $(i=0,1)$, and let $W_{2}$ be a neighborhood of $1 H$ in $G / H$ such that $\bar{W}_{2} \subset U_{2}$. Put $A_{i}=\left\{n \in N\left(K_{i}\right)^{0} ; n^{-1} \bar{W}_{i} n \subset U_{i}\right\}$. Then $A_{i}$ is an open neighborhood of the identity in $N\left(K_{i}\right)^{0}$. Let $q_{i}: \hat{N}_{i} \rightarrow N\left(K_{i}\right)^{0}$ be a finite covering such that $\hat{N}_{i}$ is a direct product $T_{i} \times G_{i}^{\prime}$. Here $T_{i}$ is a torus group and $G_{i}^{\prime}$ is a simply connected semi-simple compact Lie group. Put $\hat{K}_{i}=q_{i}^{-1}\left(K_{i}^{0}\right)$. By Assertion 6.3, there exists a closed normal subgroup $K_{i}^{\prime}$ of $\hat{N}_{i}$ such that $\hat{N}_{i}=\hat{K}_{i}^{0} \times K_{i}^{\prime}$. Let $s_{i}$ be a smooth local cross section of $q_{i}$ defined on an open neighborhood $B_{i}$ of the identity in $N\left(K_{i}\right)^{0}$. Since $\pi_{2}:\left(N(H) \cap N\left(K_{i}\right)\right)^{0} \rightarrow\left(\left(N(H) \cap N\left(K_{i}\right)\right) / H\right)^{0}$ is a fibration, there exists a smooth local cross section $t_{i}$ of $\pi_{2}$ defined on an open neighborhood $E_{i}$ of $1 H$ such that $t_{i}\left(E_{i}\right) \subset A_{i} \cap B_{i}$. 
Put $O=\left\{a \in Q_{1} ; a(i)^{-1} \in E_{i}(i=0,1)\right\}$. Then $O$ is an open neighborhood of the identity. Since $\hat{L}$ is a group homomorphism, it is enough to show that $\hat{L}$ is continuous on $O$. Let $C$ denote one of the sets $\left\{p_{i, 1 / 2}^{-1}\left(g \bar{W}_{i}\right)(i=0,1, g \in G)\right.$, $\left.\alpha^{-1}\left(g \bar{W}_{2} \times[1 / 5,4 / 5]\right)(g \in G)\right\} . \quad$ By Lemma 2.3, if $\hat{L}_{C}: O \stackrel{\mathcal{L}}{\longrightarrow} \operatorname{Diff}_{G}^{\infty}(M)^{0} \stackrel{j_{C}^{*}}{\longrightarrow}$ $C^{\infty}(C, M)$ is continuous for any $C$, then $\hat{L}$ is continuous, where $j_{C}: C \hookrightarrow M$ is an inclusion map.

First consider the case $C=p_{0,1 / 2}^{-1}\left(g \bar{W}_{i}\right)$. Let $\beta_{1}: \hat{N}_{0}=\hat{K}_{0}^{0} \times K_{0}^{\prime} \rightarrow \hat{K}_{0}^{0}$ and $\beta_{2}: \hat{N}_{0} \rightarrow K_{0}^{\prime}$ be the projection on the first factor and the second factor respectively. Let $L_{1}$ be the composition

$$
O \stackrel{r}{\longrightarrow} E_{0} \stackrel{t_{0}}{\longrightarrow} A_{0} \cap B_{0} \stackrel{\left(\xi_{g^{\prime}} q_{0} \circ \beta_{2} \circ s_{0}\right)}{\longrightarrow} C^{\infty}\left(\bar{W}_{0}, G\right) \times \operatorname{Cent}\left(K_{0}^{0}\right) \stackrel{m}{\longrightarrow} C^{\infty}\left(\bar{W}_{0}, G\right) .
$$

Here $r, \xi$ and $m$ are given by $r(a)=a(0)^{-1}, \xi_{g}\left(a_{0}\right)(x)=\xi_{a_{0}, g}(x)$ and $m(f, n)(x)$ $=f(x) \cdot n$, respectively. Put $a_{0}=\left(t_{0}^{\circ} \circ\right)(a)$ for $a \in O$. Then $\pi_{0}\left(\xi_{g, a_{0}}(x)\right)=\pi_{0}\left(a_{0}^{-1}\right)$ for $x \in \bar{W}_{0}$ and $\pi_{0}\left(\left(q_{0} \circ \beta_{2} \circ s_{0}\right)\left(a_{0}\right)\right)=\pi_{0}\left(a_{0}\right)$. Therefore $L_{1}(a) \in K_{0}$ for any $a \in O$, and $L_{1}(O) \subset C^{\infty}\left(\bar{W}_{0}, K_{0}\right)$. Let $L_{2}$ be the composition

$$
O \stackrel{r}{\longrightarrow} E_{0} \stackrel{t_{0}}{\longrightarrow} A_{0} \cap B_{0} \stackrel{q_{0}^{\circ} \beta_{1} \circ s_{0}}{\longrightarrow} N\left(H^{0}, K_{0}^{0}\right) \stackrel{\psi}{\longrightarrow} \operatorname{Diff}^{\infty}\left(D_{1 / 2}\left(V_{0}\right)\right) .
$$

By Assertion 6.5, $L_{2}$ is continuous. Let $L_{3}$ be the composition

$$
\begin{aligned}
& O \stackrel{\left(L_{1}, L_{2}\right)}{\longrightarrow} C^{\infty}\left(\bar{W}_{0}, K_{0}\right) \times \operatorname{Diff}^{\infty}\left(D_{1 / 2}\left(V_{0}\right)\right) \\
& \stackrel{\left(\rho_{1}^{*}, \rho_{2}^{*}\right)}{\longrightarrow} C^{\infty}\left(\bar{W}_{0} \times D_{1 / 2}\left(V_{0}\right), K_{0}\right) \times C^{\infty}\left(\bar{W}_{0} \times D_{1 / 2}\left(V_{0}\right), D_{1 / 2}\left(V_{0}\right)\right) \\
& \stackrel{\kappa}{\longrightarrow} C^{\infty}\left(\bar{W}_{0} \times D_{1 / 2}\left(V_{0}\right), K_{0} \times D_{1 / 2}\left(V_{0}\right)\right) \\
& \stackrel{\mu_{*}}{\longrightarrow} C^{\infty}\left(\bar{W}_{0} \times D_{1 / 2}\left(V_{0}\right), D_{1 / 2}\left(V_{0}\right)\right),
\end{aligned}
$$

where $\mu$ is given by $\mu(k, v)=k \cdot v$, and $\kappa$ is the map in Lemma 2.2. Then $L_{3}$ is continuous, and $L_{3}(a)=\tilde{h}_{2}$. Let $\gamma: A_{0} \rightarrow C^{\infty}\left(\bar{W}_{0}, U_{0}\right)$ be a map defined by $\gamma\left(a_{0}\right)(x)=a_{0}^{-1} \sigma_{0}(x) a_{0} K_{0} . \quad \gamma$ is a restriction map to $A_{0}$ of a map $\bar{\gamma}: N\left(K_{0}\right)$ $\rightarrow C^{\infty}\left(G / K_{0}, G / K_{0}\right)$ given by $\bar{\gamma}(n)\left(g K_{0}\right)=n^{-1} g n K_{0}$. Since $\bar{\gamma}$ is a continuous map, $\gamma$ is continuous. Let $L_{4}$ be the composition

$$
O \stackrel{r}{\longrightarrow} E_{0} \stackrel{t_{0}}{\longrightarrow} A_{0} \stackrel{\gamma}{\longrightarrow} C^{\infty}\left(\bar{W}_{0}, U_{0}\right) \stackrel{\rho_{1}^{*}}{\longrightarrow} C^{\infty}\left(\bar{W}_{0} \times D_{1 / 2}, U_{0}\right) .
$$

Then $L_{4}$ is continuous and $L_{4}(h)=\tilde{h}_{1} . \quad L_{C}$ is the composition

$$
\begin{aligned}
& O \stackrel{\left(L_{4}, L_{3}\right)}{\longrightarrow} C^{\infty}\left(\bar{W}_{0} \times D_{1 / 2}\left(V_{0}\right), U_{0}\right) \times C^{\infty}\left(\bar{W}_{0} \times D_{1 / 2}\left(V_{0}\right), D_{1 / 2}\left(V_{0}\right)\right) \\
& \stackrel{\kappa}{\longrightarrow} C^{\infty}\left(\bar{W}_{0} \times D_{1 / 2}\left(V_{0}\right), U_{0} \times D_{1 / 2}\left(V_{0}\right)\right) \\
& \stackrel{\left(\phi_{0}, g\right) *\left(\phi_{0, g}\right) *}{\longrightarrow} C^{\infty}\left(C, p_{0,1 / 2}^{-1}\left(g U_{0}\right)\right) \hookrightarrow C^{\infty}(C, M) .
\end{aligned}
$$

Thus $L_{C}$ is continuous.

Now consider the case $C=\alpha^{-1}\left(g \bar{W}_{2} \times[1 / 5,4 / 5]\right)$. Let $m: g \bar{W}_{2} \times N(H) / H$ 
$\rightarrow G / H$ be a map defined by $m(g H, n H)=g h H$, and let $\rho: G / H \times[1 / 5,4 / 5] \rightarrow$ $[1 / 5,4 / 5]$ be the projection on the second factor. Then $\hat{L}_{C}$ is given by the composition

$$
\begin{aligned}
& O \stackrel{i^{* \circ} \delta_{*}}{\longrightarrow} C^{\infty}([1 / 5,4 / 5], N(H) / H) \\
& \stackrel{\left(1_{g} \bar{W}_{2}\right) !}{\longrightarrow} C^{\infty}\left(g \bar{W}_{2} \times[1 / 5,4 / 5], g \bar{W}_{2} \times N(H) / H\right) \\
& \stackrel{m_{k}}{\longrightarrow} C^{\infty}\left(g \bar{W}_{2} \times[1 / 5,4 / 5], G / H\right) \\
& \stackrel{\rho \sharp}{\longrightarrow} C^{\infty}\left(g \bar{W}_{2} \times[1 / 5,4 / 5], G / H \times[1 / 5,4 / 5]\right) \\
& \stackrel{\alpha^{* \circ}\left(\alpha^{-1}\right)_{*}}{\longrightarrow} C^{\infty}\left(C, \alpha^{-1}(G / H \times[1 / 5,4 / 5])\right) \hookrightarrow C^{\infty}(C, M),
\end{aligned}
$$

where $i:[1 / 5,4 / 5] \hookrightarrow[0,1]$ is the inclusion map and $\delta: N(H) / H \rightarrow N(H) / H$ is a map given by $\delta(a)=a^{-1}$. By Lemma $2.2, \hat{L}_{C}$ is continuous.

We can see that $L_{C}$ is continuous in the case $C=p_{1,1 / 2}^{-1}\left(g \bar{W}_{1}\right)$ similarly as in the case $C=p_{0,1 / 2}^{-1}\left(g \bar{W}_{0}\right)$, and this completes the proof of Lemma 6.6.

Proof of Proposition 6.1. From Lemma 6.6, $\hat{L}\left(Q_{1}\right)$ is contained in $\operatorname{Diff}_{G}^{\infty}(M)_{0}$. Then, by the definition, $\hat{L}\left(Q_{1}\right)$ is contained in $S$, and $\hat{L}=L^{-1}$. Combining Lemma 4.5, Proposition 4.6 and Lemma 6.6, $\hat{L}: S \rightarrow Q_{1}$ is an isomorphism between topological groups, and this completes the proof of Proposition 6.1 .

Proof of Theorem. By Corollary 3.6, $\operatorname{Diff}_{G}^{\infty}(M)_{0}$ has the same homotopy type as Ker $P$. Combining Lemma 5.1, Lemma 5.2 and Proposition 6.1, Ker $P$ has the same homotopy type as $Q_{0}$. Note that $Q_{0}$ has the same homotopy type as the path space $\Omega\left(N(H) / H ;\left(N(H) \cap N\left(K_{0}\right)\right) / H,\left(N(H) \cap N\left(K_{1}\right)\right) / H\right)_{0}$. This completes the proof of our Theorem.

\section{§7. Concluding Remarks}

From our Theorem, we have the following

Corollary 7.1. (1) If $K_{0}=K_{1}=G$, then $\operatorname{Diff}_{G}^{\infty}(M)_{0}$ has the same homotopy type as $(N(H) / H)^{0}$.

(2) If $N(H) / H$ is a finite group, then $\operatorname{Diff}_{G}^{\infty}(M)_{0}$ is contractible.

Remark 7.2. In K. Abe and K. Fukui [1], we have proved that $\operatorname{Diff}_{G}^{\infty}(M)_{0}$ is perfect if $M$ is a $G$-manifold with one orbit type and $\operatorname{dim} M / G \geq 1$. But, by using Proposition 3.1, we can see that $\operatorname{Diff}_{G}^{\infty}(M)_{0}$ is not perfect in the case $M / G=[0,1]$. 


\section{References}

[1] Abe, K. and Fukui, K., On commutators of equivariant diffeomorphisms, Proc. Japan Acad., 54 (1978), 52-54.

[2] Abraham, R., Lectures of Smale on Differential topology, Mimeographed Notes, Columbia Univ., 1962.

[3] Bredon, G., Introduction to Compact Transformation Groups, Academic Press, New York-London, 1972.

[4] Cerf, J., Topologie de certains espaces de plongements, Bull. Soc. Math. France, 89 (1961), 227-380.

[ 5] Dieudonné, J., Foundations of Modern Analysis, Academic Press, New York-London, 1960.

[6] Hirsch, M., Differential Topology, Springer-Verlag, New York-Heidelberg-Berlin, 1976.

[ 7] Hsiang, W. C. and Hsiang, W. Y., Classification of differentiable actions on $S^{n}, R^{n}$ and $D^{n}$ with $S^{k}$ as the principal orbit, Ann. of Math., 82 (1965), 421-433.

[8] Pontrjagin, L., Topological Groups, Princeton Univ. Press, Princeton, New York, 1946.

[9] Yokota, I., Groups and Representations (in Japanese), Shokabo, 1973. 